Théologiques

Théologiques

\title{
Ambiguïtés et désir en Qohélet 6,7-9
}

\section{Jean-Jacques Lavoie}

Volume 22, numéro 2, 2014

URI : https://id.erudit.org/iderudit/1035691ar

DOI : https://doi.org/10.7202/1035691ar

Aller au sommaire du numéro

\section{Éditeur(s)}

Faculté de théologie et de sciences des religions, Université de Montréal

\section{ISSN}

1188-7109 (imprimé)

1492-1413 (numérique)

Découvrir la revue

\section{Citer cet article}

Lavoie, J.-J. (2014). Ambiguïtés et désir en Qohélet 6,7-9. Théologiques, 22(2), 179-215. https://doi.org/10.7202/1035691ar

\section{Résumé de l'article}

L'auteur présente un état de la recherche des critiques textuelle et des sources de Qohélet 6,7-9 et propose une analyse structurelle et littéraire de ces versets. Puis, il poursuit son enquête en montrant que les ambiguïtés de Qohélet 6,7-9 ne sont pas purement accidentelles. Au contraire, elles font partie d'une stratégie rhétorique qui vise à inciter le lecteur à éprouver différentes lectures. d'utilisation que vous pouvez consulter en ligne.

https://apropos.erudit.org/fr/usagers/politique-dutilisation/ 


\title{
Ambiguiités et désir en Qohélet 6,7-9
}

\author{
Jean-Jacques LAvoiE* \\ Sciences des religions \\ Université du Québec à Montréal (Canada)
}

Mon intention, dans cet article, est d'expliquer Qo 6,7-9, un texte reconnu pour être l'un des passages énigmatiques du livre (SchwienhorstSchönberger 2004, 355). De l'avis d'Ehrlich, cité par Ellermeier (1963, 2), le v. 8 b est à ce point obscur que seul Dieu sait ce qu'il signifie! Pour expliquer ces trois versets qui posent divers problèmes aux exégètes, je proposerai d'abord non pas une traduction, mais des traductions possibles du texte. Puis, j'analyserai quelques variantes textuelles qui suscitent certains problèmes de traduction et donc d'interprétation. Je confronterai par la suite les principaux résultats issus de l'approche diachronique et de l'hypothèse des citations à une critique structurelle. Je terminerai mon enquête par une critique littéraire du texte, c'est-à-dire une analyse philologique, syntaxique et sémantique de chacun de ces trois versets. L'application de ces diverses méthodes me permettra non seulement de valider et d'infirmer certaines interprétations existantes, mais aussi d'en proposer de nouvelles, qui sont tantôt complémentaires et tantôt en contradiction avec celles déjà défendues par mes prédécesseurs.

\section{Traduction}

V. 7: Tout le labeur de l'être humain est pour sa bouche/sa faim/son appétit, mais pourtant son/l'être-de-désir n'est pas rempli.

* Jean-Jacques Lavoie est professeur titulaire au Département des sciences des religions de l’Université du Québec à Montréal. Spécialiste des textes sapientiaux de la période du Second Temple, il a publié, en 2013-2014, des articles sur Proverbes, Cantique des cantiques et Qohélet, dans les revues suivantes: Laval théologique et philosophique, Science et Esprit, Theoforum, Sciences religieuses/Studies in Religion, Rivista Biblica, Estudios biblicos, Revue des sciences religieuses et Ephemerides Theologicae Lovanienses. 
V. 8: Car/Vraiment, quel avantage a le sage sur l'insensé ? Quel [avantage] a/qu'a le pauvre/l'humble qui sait marcher/se conduire devant/contre la vie/les vivants?

V. 9: Bonne une vision des yeux plus que la poursuite du désir/plus qu'un être-de-désir qui s'en va; cela aussi est absurdité et pâturage/poursuite de vent/souffle.

\section{Critique textuelle}

\subsection{Verset 8}

Au v. 8a, un manuscrit de Qumran (4QQoha , colonne II, fragments 1 ii,3-6 i) a kmb ywtr, au lieu de ky mb ywtr du texte massorétique (Puech 2001, 148). Selon Muilenberg, l'emploi de $k$, qui est une simple variante de $k y$, est la trace de l'orthographe phénicien dans lequel était omis la mater lectionis (Muilenberg 1954, 25). Puech abonde dans le même sens, mais n'écarte pas la possibilité d'une haplographie à partir d'un texte rédigé en scriptio plena, erreur répandue chez les scribes qui copiaient les textes bibliques (Puech 2001, 149). Au contraire, Goldman est d'avis que cette variante vise probablement à donner un avantage au sage $(2004,88)$. Est-ce à dire que l'on peut donner à l'expression $\mathrm{kmb}$ un sens adverbial et traduire le texte de Qumran comme suit: "Que d'avantage pour le sage sur l'insensé! »? Il est vrai que le mot $m h$ peut avoir un sens adverbial (Joüon 1982, $446=143 \mathrm{e}$ ), mais peut-on en dire autant de l'expression $k m b$ ? Ou faut-il donner à kmh son sens habituel de "combien? " (voir Gn 47,8; Ps 78,40; Za 2,6; etc.): «Combien d'avantage a le sage sur l'insensé ? " À mon avis, les interprétations de Muilenberg, Puech et Goldman sont fragiles. Premièrement, du point de vue de la graphie, il est peu probable qu'un archaïsme phénicien (absence de la mater lectionis dans la conjonction $k y$ ) ait encore été en usage à l'époque tardive de Qumran. Deuxièmement, l'hypothèse d'une haplographie est peu vraisemblable, car la lettre $y$ n'apparaît qu'après le mot $m h$. Troisièmement, du point de vue grammatical, l'expression $k m b$ peut très bien être rendue par une question. Bref, il serait imprudent de corriger le texte massorétique à partir du seul manuscrit de Qumran.

Par ailleurs, le traducteur de la version syriaque a bel et bien compris le v. 8 a comme une affirmation positive en faveur du sage: $m t ̦ l$ d'yt ywtrn' lhkym' (Lane 1979, 10), "il arrive qu'il y ait un profit pour le sage ». Que la version syriaque soit dépendante (Goldman 2004, 88) ou non (Kamenetzky 1904, 219) de la Septante, il est intéressant de noter que les 
Codices Vaticanus et Sinaïticus de la Septante, qui n'ont pas été retenus par Rahlfs $(1935,249)$ et Vinel $(2002,140)$, n'ont pas le pronom interrogatif tis: hoti perisseia, littéralement "parce qu'un avantage». Dans les versions dites d'Aquila et de Théodotion, le pronom interrogatif tis est certes présent, mais il est accompagné d'un astérisque dans la version syrohexaplaire du Codex Ambrosianus datant du IX ${ }^{\mathrm{e}}$ siècle (Field 1875, 391). Par conséquent, il est légitime de se demander si ce pronom interrogatif faisait partie du texte grec original. Quoi qu'il en soit de la réponse à cette question, j'estime que les lectures qui visent à faire du v. 8 a une affirmation en faveur du sage peuvent difficilement correspondre au texte hébreu originel, car le livre de Qo, on le verra, souligne à maintes reprises le fait que le sage n'a aucun avantage sur l'insensé. Par conséquent, ces lectures, qui sont peut-être fondées sur Qo 2,3, visent probablement à amoindrir le caractère scandaleux du texte de Qo.

Au v. 8b, le traducteur de la Septante a rendu le texte comme suit: dioti ho penēs oiden poreuthēnai katenanti tēs zōess (Rahlfs 1935, 249). Selon la version syrohexaplaire (Field 1875, 391), les versions d'Aquila et de Théodotion ont également le mot dioti. Vinel comprend ce mot comme une conjonction causale: "c'est pourquoi le pauvre sait aller en face de la vie» $(2002,140)$. Goldman n'exclut pas que la conjonction dioti puisse avoir le sens d'une interrogation directe (2004, 88). C'est à mon avis peu probable. En effet, le mot dioti a un sens causal dans le seul autre emploi du livre de Qohélet, en 2,15f, un ajout du traducteur grec: dioti aphrōn ek perisseumatos lalei, "parce qu'un insensé parle avec excès ». Goldman est également d'avis que la version syriaque peut avoir un sens interrogatif ou causal $(2004,88)$. À mon avis, la version syriaque ne peut être interprétée comme une interrogation directe. Il est vrai qu'elle a le pronom interrogatif lmn', "pourquoi ?", mais comme il est suivi du pronom relatif $d$, il joue le rôle d'un pronom démonstratif $($ Costaz 2003, 72 = no 309): "c'[est] pourquoi ». La version syriaque a donc le même sens que la Septante.

Par ailleurs, la version syrohexaplaire $(m t ̣ l m n$ ') témoigne d'une autre leçon grecque: dia ti, "à cause de quoi » (Field 1875, 391; Goldman 2004, 88). Quant à la version de Symmaque, elle a bel et bien une interrogation, mais avec l'ajout de la particule de qui n'a pas d'équivalent dans le texte hébreu : ti de tô ptōchō, «mais pourquoi le mendiant? ». Pour ce qui est de Jérôme, dans la Vulgate, il omet de traduire le verbe $y d^{`}$ et ajoute la conjonction nisi après le mot pauvre: et quid pauper nisi ut pergat illuc ubi est vita $(1957,155)$, "et qu'a le pauvre, sinon qu'il va là où est la vie?». 
Que doit-on conclure de ces différentes versions? Il est impossible de justifier une correction du texte hébreu à partir de la Vulgate, car il est évident que Jérôme cherche à établir un parallèle entre les v. $8 \mathrm{~b}$ et $8 \mathrm{a}$. Quant à la variante de la version de Symmaque, elle est mineure et vise surtout à rendre le texte plus coulant. Peut-on en dire autant des versions grecques de la Septante, d'Aquila et de Théodotion et de la version syriaque? Selon Kamenetzky (1904, 220) et Lange (1991, 135), ces versions supposent l'hébreu $l m h$, le $l$ ayant pu disparaître par haplographie à cause de sa présence à la fin du mot précédent $(h k y l)$. À mon avis, Goldman a raison d'affirmer qu'aucune de ces versions ne suppose nécessairement un texte hébreu différent $(2004,88)$.

Pourtant, force est de constater que les exégètes proposent diverses corrections du texte hébreu du v. $8 \mathrm{~b}$, particulièrement de l'expression $m b$ l'ny, littéralement «quoi pour le pauvre? ». Par exemple, voulant faire du v. 8 b la réponse à la question du v. 8 a (quel est l'avantage du sage sur l'insensé ?), Scott propose de lire mibbil'adē yōde' $a$, "à part sa connaissance " ou "sauf qu'il sait ", c'est-à-dire: seulement en sachant comment se conduire soi-même durant sa vie $(1965,231)$. Bien qu'il ignore le commentaire de Scott, Fischer défend la même correction $(1997,73)$. Celle-ci est pourtant injustifiable, car elle suppose des modifications majeures: la disparition de trois consonnes, un nouveau découpage des mots et une nouvelle vocalisation. La correction de Hertzberg, qui propose de lire mēhe ‘̄ầ layyôde' $a$, "sur le pauvre qui sait..." (1963, 128 et 130), n'est pas davantage justifiée, car elle suppose une autre vocalisation du mot $m b$ et un déplacement arbitraire du $l$ qui précède le mot ' $n y$ devant le verbe

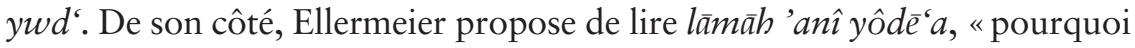
moi je comprends?" $(1967,12)$. Pour défendre cette correction, qui a été adoptée par Galling $(1969,104)$, Loader $(1979,85-86)$ et Lange (1991, 135 et 138), deux arguments sont présentés. Le premier, c'est la confusion fréquente entre le 'et le '. Le second, c'est l'absence du mot 'ny pour désigner le pauvre dans le reste du livre. On invoque le fait que Qohélet emploie plutôt le mot mskn pour désigner le pauvre $(4,13$ et 9,15). La valeur du second argument est faible, car s'il fallait suspecter tous les mots qui n'apparaissent qu'une seule fois en Qo, c'est 271 mots qu'il faudrait remettre en question ${ }^{1}$ ! En outre, Qo emploie aussi le mot $r$ š pour désigner le pauvre $(4,14 ; 5,7)$. Enfin, cette correction, comme toutes les autres, est

1. Sur ces 271 mots, 27 sont des hapax legomena. Ce calcul est effectué à partir de la liste des mots de Qo présentée par Delsman (2000, 138-147 et 243-267). 
d'autant plus injustifiée qu'elle n'a aucun appui textuel dans les anciennes versions. Beaucoup plus modéré, Murphy propose simplement de vocaliser le $l$ qui précède le mot ' $n y$ avec un šewa' au lieu du segôl du texte massorétique, lisant ainsi le mot 'ny sans article (Murphy 1992, 48). Cette correction me semble inutile, car il est bien connu que l'emploi de l'article en Qo est irrégulier (Isaksson 1987, 147; Weeks 2012, 189-190). En définitive, il est donc préférable de maintenir le texte massorétique.

\subsection{Verset 9}

Au lieu du verbe $h l k$, "aller ", Ginsberg suggère de lire le verbe $m l w$ ', "être plein », et propose la traduction suivante: «qu'un gosier plein» (1960, 142). Il justifie cette correction en affirmant que le verbe hlk provient de son emploi au verset précédent. Cette correction n'a aucun appui dans les anciennes versions. Par exemple, la Septante suppose bel et bien le verbe $h l k$. Toutefois, le traducteur a rendu l'infinitif construit du texte massorétique par un participe: huper poreuomenon psuchē, "plus qu'allant à l'âme» (Rahlfs 1935, 249). Goldman se demande si cette traduction, qui est confirmée par celle de Jérôme dans son commentaire de l'Ecclésiaste (1959, 299), ne vise pas à éviter l'idée de la pérégrination de l'âme (2004, 88). Ce n'est pas impossible. Toutefois, je serais plutôt enclin à attribuer cette divergence à la liberté du traducteur. En effet, Vinel a déjà remarqué que le traducteur grec n'est pas systématique dans la façon dont il rend le temps des verbes (2002, 54-55). Deux exemples supplémentaires à ceux qu'elle donne vont permettre de confirmer mon propos. Premièrement, au v. 8, le verbe $y w d^{\prime}$, au participe, est rendu par l'infinitif oiden. Or, cette traduction d'un verbe au participe par un verbe à l'indicatif apparaît plus de quarante autres fois en Qo (avec le verbe $y d^{\prime}: 2,19 ; 3,21 ; 6,10.12$; $8,1.12 ; 11,6$; avec le verbe $h l k: 1,4.6[2 \mathrm{x}] .7 ; 2,14 ; 3,20 ; 6,6 ; 9,10 ; 10,3$; 12,5 ; avec le verbe $b^{\prime}: 1,4.5$; etc.). Deuxièmement, les verbes au qal infinitif sont parfois rendus par des verbes à l'indicatif (lbrm - diakrinei: 3,18; ntwn-edōka: 8,9; r'h-eidon: 9,11).

Quoi qu'il en soit de l'interprétation de Goldman, les anciennes versions confirment l'emploi du verbe hlk à l'infinitif. En effet, Symmaque emploie le verbe hodeuein: beltion problepein è hoduein autareskeia (Field $1875,391)$, "mieux vaut regarder d'avance que de faire route avec suffisance ». Quant au substantif $h l k t$ ', "marche », "promenade ", de la version syriaque, il est l'équivalent du verbe à l'infinitif. En somme, il convient de maintenir le texte massorétique et de lire le verbe hlk à l'infinitif. 


\section{Approche diachronique et hypothèse des citations}

Au début du $\mathrm{xx}^{\mathrm{e}}$ siècle, il était commun d'attribuer Qo 6,7-9 à divers rédacteurs (Podechard 1912, 358-360; Buzy 1946, 239-240). Par la suite, ce genre d'hypothèse a été progressivement délaissé. Par exemple, Alonso Diaz juge que les v. 7 et 9b sont des ajouts $(1967,78)$, tandis que Rankin limite l'intervention du glossateur au seul v. 7 (1956, 62). Plus circonspects, Zimmermann $(1973,134)$ et Bottéro $(1986,239)$ sont d'avis que le v. 7 est peut-être une interpolation. Par ailleurs, Bottéro juge que les v. 8-9 sont des versets erratiques et se demande si le v. 9 n'est pas une glose du v. 7 qui serait authentique. De son côté, Fischer propose de lire le v. 9b comme une marque éditoriale du rédacteur qui étaye l'interprétation globale donnée par 1,2 et 12,8, laquelle encadre l'ensemble du livre (1997, $13,59)$. Fox se contente d'affirmer que le v. 8 n'est pas à sa place puisqu'il interrompt le lien entre les v. 7 et 9 (Fox 2004, 41). En 1999, deux auteurs renouent avec l'idée que le livre de Qo aurait été rédigé par plus d'un rédacteur et en plusieurs étapes. Très enclin à la spéculation, Rose, qui postule trois étapes rédactionnelles dans le livre de Qo, attribue les v. 7-8.9b au disciple de Qohélet et le v. 9a au théologien-rédacteur (Rose 1999, 316-318 et 382-383). Selon lui, le disciple, qui est le responsable de la première relecture du livre de Qo, cherche à donner davantage de volume au livret de son maître $(1999,282)$, tandis que le théologien-rédacteur, qui est le responsable de la troisième étape rédactionnelle, cherche plutôt à donner au livre un ton plus orthodoxe et traditionaliste (1999, 181, 221 et 232). Tout aussi débordante d'imagination, Brandscheidt, qui retrace quatre étapes rédactionnelles dans le livre de Qo, redistribue les trois versets comme suit: le v. $7 \mathrm{a}$ appartient au texte primitif, tandis que les v. 7b-9 sont de la main du deuxième rédacteur $(1999,129-130,188$, 278 et $459-462$ ).

Tout aussi fantaisiste, Coppens est d'avis qu'il n'y a qu'un seul auteur derrière le livre de Qo, mais il précise que celui-ci a entrepris la rédaction de son œuvre en quatre étapes bien distinctes qui correspondent à quatre moments précis de sa vie. Au sujet des v. 7-9, il estime qu'ils font partie de la strate IIB de l'écrit fondamental, dans laquelle Qohélet invoque une série de constats faits à partir de ce qu'il a observé autour de lui (1979, 288-289).

L'interprétation de Perry est tout aussi inutilement alambiquée, voire arbitraire, puisqu'il imagine que le livre est un dialogue entre Qohélet, le sage et le roi (Qo 6,7a.8.9b), et son présentateur plus orthodoxe et tolérant (Qo 6,7b.9a) (1993, 116). 
Bien entendu, rien ne nous autorise à voir un dialogue dans ces versets. Il suffit de comparer le livre de Job avec le livre de Qo pour se rendre à l'évidence que ce dernier n'a rien de commun avec un dialogue entre deux interlocuteurs.

Depuis déjà plusieurs années, l'interprétation selon laquelle le livre de Qo serait truffé de citations a pris le relais de l'hypothèse des sources. Autrement dit, le mot glose a été remplacé par le mot citation. Toutefois, le problème posé par cette nouvelle hypothèse est le même que celui de la critique des sources, c'est-à-dire celui de la délimitation des citations. En effet, d'aucuns voient des citations dans le v. 7 (Reichert et Cohen 1946, 147; Steinmann 1955, 79; Loewenclau 1986, 331; Michel 1989, 150; Bonora 1992, 108; etc.), le v. 7b (Perry 1993, 116), le v. 9a (Scott 1965, 232; Lauha 1978, 107; 117; Fischer 1986, 78) ou encore dans les v. 7 et 9a (Tamez 2000, 89; Whybray, 1981, 437-438, avec hésitation).

Bien entendu, il n'y a pas que la délimitation des citations qui pose des difficultés. Leur fonction est tout aussi problématique. Par exemple, selon Michel, le v. 7 est une citation de l'interlocuteur qui réagit à ce que Qohélet a dit au v. 3, mais cette citation est aussitôt commentée de manière critique par Qohélet aux v. 8-9 (Michel 1988, 147; 1989, 150-151). Au contraire, d'autres sont d'avis que le v. 7 est un dicton populaire que Qohélet cite à l'appui de la conclusion qu'il va tirer (Steinmann 1955, 79; Gordis 1971, 260). Enfin, Reichert et Cohen jugent plutôt que la citation du v. 7 sert à prouver la véracité du v. 5 (1946, 147). La fonction de la soi-disant citation du v. 9a ne fait pas davantage l'unanimité. Par exemple, Fischer et Tamez sont d'avis que cette citation exprime la pensée de Qohélet (Fischer 1986, 78; Tamez 2000, 89-90). Au contraire, Murphy et Pinker sont plutôt d'avis - mais pour des raisons différentes, sur lesquelles je reviendrai - que Qohélet reprend ici un proverbe avec lequel il est en désaccord, d'où le verdict final au v. 9b (Murphy 1992, 54; Pinker 2009, 225). Déjà au XVIII siècle, Yerushalmi attribuait le v. 9a au fou, lequel était réfuté au v. $9 b(1988,140)$. Force est de constater que l'identification et la fonction des citations comme celles des gloses dépendent du genre de message que l'on veut bien voir dans ce texte.

\section{Critique structurelle}

La délimitation de la péricope varie d'un exégète à l'autre: 6,3-9 (Stephanus 1993, 78), 6,6-9 (Ogden 1987, 93), 6,6-10 (Michaud, 1987, 169), 6,7-8.9 (Loader 1979, 85-87), 6,7-12 (Walton, 2006, 162), etc. À mon avis, il y a 
de bonnes raisons qui permettent d'affirmer que la péricope se termine au v. 9. Premièrement, l'expression $r^{6} w t$ rwh conclut toujours une réflexion $(1,14 ; 2,17 ; 4,4)$ ou une péricope $(2,11.26 ; 4,6)$; c'est également le cas de l'expression apparentée $r^{6} y w n$ rwh en Qo 1,17 et 4,16. Comme il s'agit du dernier emploi de l'expression $r^{6} w t r w h$, il est fort probable qu'elle conclue une péricope. Deuxièmement, à la suite des massorètes, il convient de reconnaître que le v. 10 est le milieu du livre. Plus précisément, Qo 6,10-12 constitue une unité de transition qui divise le livre en deux grandes parties: Qo 1,1-6,9 et 7,1-12,14 (Lavoie 2014a, 11-12).

S'il ne fait pas de doute que le v. 9 clôt une péricope, voire la première partie du livre, il est plus difficile de dire où cette péricope commence et quelle est sa relation avec ce qui précède. Selon Reinert, Qo 6,5-9a constitue une petite péricope à l'intérieur d'une section qui va de 5,12 à 6,12 (2010, 154). Plus précisément, il est d'avis que Qo 6 doit se lire comme suit: observation $(6,1 \mathrm{a})$, description $(6,1 \mathrm{~b} .2 \mathrm{a})$, résultat $1(6,2 \mathrm{~b})$, explication $1(6,3)$, résultat $2(6,4)$, explication $2(6,5-9 a)$, résultat $3(6,9 b)$, explication $3(6,10-12)(2010,156)$.

Lange rattache également 6,7-9 à ce qui précède, mais délimite autrement les petites unités: une introduction $(6,1)$, trois descriptions du mal présentées sous forme de climax: un seul homme $(6,2)$, cent enfants $(6,3)$, deux mille ans $(6,6)$ et des remarques qui signalent les conséquences de l'observation (6,7-9) (1991, 136-137). À mon avis, cette délimitation a le mérite de montrer qu'il y a une sorte de crescendo entre les v. 1 et 6 et que les petites unités sont liées entre elles. Toutefois, une analyse plus serrée indique que les v. 1-2 constituent une première unité construite sous la forme d'un chiasme (Lavoie 2011, 167-168) et que les v. 3-6 forment une deuxième unité délimitée par la reprise d'un vocabulaire précis, soit celui de la vie (hyh:6,3.6), de l'abondance et de la longévité (cent, nombreuses années, deux fois mille ans: 6,3.6), de l'absence de bonheur $(t w b h: 6,3.6)$ et de la mort (sépulture et lieu unique: 6,3.6). Quant aux v. 7-9, ils sont encadrés par le mot $n p$ š qui est jumelé à deux parties du corps: bouche - npš en 6,7 et yeux - npš en 6,9a. Plus précisément, le v. 7 est une première sentence qui évoque les deux unités précédentes. En effet, ce verset rappelle d'abord 6,2, d'une part, par la reprise du mot ' $d m(6,2.7)$ et, d'autre part, par l'emploi du mot bouche $(p h)$ qui n'est pas sans lien avec le verbe manger (' $k l: 6,2$ [2x]). Ce v. 7 rappelle aussi 6,3, car dans les deux cas il est question de non satisfaction, une première fois avec le verbe śs $(6,3)$ et une seconde fois avec le verbe $m l^{\prime}$ (6,7). La répétition du mot l' qui exprime la négation assure aussi une continuité entre les trois petites unités 
(6,2.3 [2x].5 [2x]. 6 [2x].7). Le v. 8 est formé de deux questions, dont le sens et le lien ne peuvent être déterminés que par une analyse méticuleuse du vocabulaire. Du point de vue de la forme, ces deux questions rappellent la question rhétorique du v. 6b. Par ailleurs, du point de vue du vocabulaire, on peut signaler la reprise du verbe $h l k(6,4.6)$ et de la racine hyh (hyb en 6,3.6 et hyym en 6,8). Enfin, le v. 9a est constitué d'un dit de préférence, comme au v. 3 , et d'un verdict final $(6,9 \mathrm{~b})$. Au plan lexical, la reprise de la racine $r^{\prime} h(6,1.5 .6 .9)$ et du mot $h b l(6,2.4 .9)$ crée une continuité entre le v. 9 et les deux unités précédentes. En outre, le dit de préférence du v. 9a fait écho au v. 1, car, contrairement à son emploi aux v. 5 et 6 , la racine $r$ 'b n'est pas précédée de la négation. Si l'on ajoute à cela la reprise des mots țwb (b) (6,3 [2x].6.9), hlk (6,4.6.8.9) et $n p \check{s}(6,7.9)$, le v. 9 récapitule l'ensemble des versets précédents du chapitre 6 , car seule l'expression $r^{6} w t$ t $w$ h est unique. Enfin, la répétition du mot $n p s ̌ s(6,2.3 .7 .9)$ et l'emploi du verbe 'wh, "désirer» $(6,2)$, indiquent que le désir constitue un thème central de 6,1-9. En définitive, il n'y a donc pas lieu de trop isoler 6,7-9 des six versets qui le précèdent. C'est par exemple ce que fait Laurent qui estime que Qo 5,9-6,6 constitue une grande section qui a en commun un vocabulaire et un thème, celui des biens matériels, qui sont tous deux absents de 6,7-9 $(2002,14)$.

Par ailleurs, parmi les exégètes qui rattachent 6,7-9 à ce qui précède, nombreux sont ceux qui lient cette péricope non pas à 6,1-6, mais à 5,911 ou à 5,7-11. En effet, Fredericks considère que Qo 5,9-6,9 est construit sous la forme, d'une part, d'une structure chiastique et, d'autre part, d'une structure parallèle $(1989,17-35$; 2010, 148-149). Selon lui, le chiasme se présente comme suit: A et $\mathrm{A}^{\prime}$ : limitation à la satisfaction $(5,9$ 11 et 6,7-9); B et B': aller et retour dans les ténèbres (5,12-16 et 6,3-6); C et C': bénédiction de Dieu ou malédiction de Dieu ? (5,17-19 et 6,1-2). En ce qui concerne 6,7-9 et 5,9-11, Fredericks fonde le parallélisme sur les reprises suivantes: A et $\mathrm{A}^{\prime}$ : les limites à la satisfaction $(5,9.11$ et 6,7.9); B et B': le caractère temporaire des possessions (5,9.10 et 6,9); C et C': la question sur l'avantage $(5,10$ et 6,8$)$; D et D': l'idée de se réjouir de ce que l'on peut voir $(5,10$ et 6,9$)$. Quant à la structure parallèle, elle est définie comme suit: une introduction $(5,9-11)$ est suivie de deux sections $(5,12-19$ et $6,1-9)$ dans lesquelles sont exploités trois thèmes: le mal (5,12-13a et 6,1-2), l'engendrement (5,13b-15a et 6,3-6) et la question de l'avantage provenant du travail (5,15b-19 et 6,7-9). Pour l'essentiel, cette structure a été reprise par quelques exégètes (Vilchez Lindez 1994, 281 ; Anaya Luengo 2007, 170-171; Fischer 1999, 51-52). Ce dernier affirme 
même que cette longue section fait partie des passages les mieux structurés de tout le livre de Qo.

Ignorant l'article de Fredericks, pourtant déjà publié depuis huit ans, Krüger est également d'avis que 5,9-6,9 constitue une grande section (1997, 59-60). Toutefois, il estime que celle-ci est plutôt construite sous la forme d'un chiasme avec une pointe émergente: A et A': trois proverbes (5,9-11 et 6,7-9); B et B': cas négatif: le mal $(5,12-16$ et $6,1-6)$; C: cas positif: le bonheur $(5,17-19)$. La même année, Fischer propose la même structure sous forme de chiasme (1997, 74 et 253). En 2012, SchwienhorstSchönberger abandonne son ancienne interprétation, selon laquelle 6,7-9 répondait à 4,1-6 $(2004,288)$ — interprétation reprise la même année par Asurmendi $(2012,82)$ ! - , et adopte cette analyse structurelle de Krüger et de Fischer, mais sans pour autant citer l'un ou l'autre de ces auteurs (Birnbaum et Schiwienhorst-Schönberger 2012, 115). Poussant plus loin son analyse, Krüger souligne aussi la correspondance entre 5,9-11 et 5,126,6: A et A': pas de satisfaction, avec le verbe śb 'précédé de la négation $l$ ' (5,9 et 6,3-6); B et B': consommation, avec le verbe ' $k l(5,10$ et $6,1-2)$; C et $C^{\prime}$ : homme riche et travailleur $(5,11$ et $5,12-16)(1997,60)$. On notera que cette divergence entre Fredericks et Krüger ne les empêche pas de s'entendre quant à la correspondance entre 6,7-9 et 5,9-11.

S'inspirant de l'analyse de Fredericks, Seow est plutôt d'avis que la section commence avec 5,7 et que celle-ci est construite sous la forme d'un chiasme avec une pointe émergente: A et A': 5,7-11 et 6,7-9; B et B': 5,1216 et 6,3-6; C et C’: 5,17-18 et 6,1-2; D : 5,19 (1997, 217). Kamano fait sienne cette analyse de Seow $(2002,136-137)$. Selon cette analyse, il y a donc également une correspondance entre 6,7-9 et 5,9-11, mais celle-ci est élargie à 5,7-8. Selon Seow, quatre thèmes sont communs aux deux unités: le pauvre en 5,7 et l'affligé en 6,8; la non satisfaction en 5,9 et 6,7; la question de l'accomplissement en 5,10 et de l'avantage en 6,8 et, enfin, la vue de ses yeux en 5,10 et la vue des yeux en 6,9.

À mon avis, le lien entre le pauvre ( $r \grave{s}$ ) et l'affligé ('ny) est trop ténu pour être retenu. En outre, Qo 5,9-11 forme une petite unité construite de manière concentrique autour du v. 11a (Lavoie 1992, 339). Quant aux autres soi-disant liens qui rattachent 6,7-9 à 5,9-11, ils sont plutôt thématiques et ils reposent sur des interprétations discutables. Premièrement, le thème de l'insatisfaction n'est guère concluant, car il apparaît aussi en 6,3-6 et, qui plus est, avec le même verbe (śb' $: 5,9$ et 6,3 ). Deuxièmement, il est abusif d'affirmer que 6,9 traite du thème des possessions temporaires. En effet, les mots argent $(k s f)$, richesse ( $h m w n)$ et revenu ( $t b w$ 'b), qui sont 
employés en 5,9, ne sont aucunement repris en 6, 9. Quant au mot possesseur $\left(b^{`} l\right)$, il est employé aussi bien en 5,10 qu'en 5,12 qui fait partie d'une autre péricope. Troisièmement, le thème de l'avantage n'est guère plus concluant, d'une part, parce que les deux questions en 5,10 et 6,8 ne sont pas formulées avec le même terme ( $m b$ kšrwn en 5,10 et $m b$ ywtr en 6,8) et, d'autre part, parce que la question de l'avantage ( $m b$ ytrwn) apparaît également en 5,15. Quatrièmement, le thème de la réjouissance de ce que l'on peut voir $(5,10$ et 6,9$)$ est certes fondé sur la reprise de la racine $r$ 'b et du mot ' $y n$. Toutefois, lors de mon analyse du vocabulaire, j'aurai l'occasion de montrer que les deux expressions n'ont aucunement le même sens. Il ne faut donc pas se laisser prendre au piège de la confusion entre connotation et dénotation. En définitive, il apparaît plus prudent d'essayer de comprendre 6,7-9 en fonction de son contexte immédiat, c'est-à-dire Qo 6,1-6.

Bien entendu, cette critique structurelle ne permet pas, à elle seule, de comprendre la signification de Qo 6,7-9. Il me faut donc poursuivre mon étude par une analyse philologique, grammaticale et sémantique de chacun des mots de ces deux versets.

\section{Critique littéraire}

Ma critique textuelle permet de lire le texte massorétique tel quel, sans lui imposer aucune correction, tandis que ma critique structurelle permet de comprendre le texte tel qu'il se donne à lire maintenant, sans avoir à imaginer l'intervention d'un rédacteur quelconque. En outre, ma critique structurelle indique bien que les v. 7-9, même s'ils ne sont pas sans lien avec ce qui précède, forment une petite unité autonome. En toute rigueur de méthode, il me faut maintenant analyser chacun des mots de ces trois versets afin de pouvoir bien comprendre leur signification.

\subsection{Verset 7}

La racine ' $m l$ est un terme clé du livre de Qo, car elle apparaît 35 fois: on y trouve 22 fois le substantif ' $\bar{a} m \bar{a} l$, huit fois le verbe 'àmal et cinq fois l'adjectif 'àmēl. Il est vrai que la racine ' $m l$ est polysémique dans les langues anciennes du Proche-Orient ainsi qu'en hébreu biblique (Lavoie 2004, 62-65). Toutefois, son emploi dans le livre de Qo n'est aucunement polysémique. Contrairement à ceux qui traduisent le mot ' $m l$ en 6,7 par «revenu» (Foresti 1980, 429) ou par «gain» (Steinmann 1955, 78 et la NJPS commentée par Fox 2004, 41), je suis donc d'avis que ce mot désigne 
le travail dans ce qu'il a de plus pénible et fatiguant, d'où la traduction par "labeur", mot qui dérive du latin labor, " peines ». C'est ce dont témoigne déjà la traduction de la Septante. En effet, contrairement au reste de la Bible où le mot 'āmāl est rendu par six termes différents, le traducteur grec du livre de Qo l'a systématiquement rendu par mochthos, "peine ", "travail », "fatigue ». Ce caractère pénible du travail est bien confirmé en Qo, puisqu'il est associé avec les mots suivants: "absurdité et pâturage de vent " $(2,11 ; 4,4)$, "absurdité et grand malheur» $(2,21)$, "absurdité et mauvais souci » $(4,8)$, "absurdité » $(2,19 ; 9,9)$, "pâturage de vent » $(4,6)$, "pâturage » $(2,22)$, «du vent » $(5,15)$, «mal douloureux » $(5,15)$, «haïr » $(2,18)$, "être fatigué » $(10,15)$, "désespérer » $(2,20)$, "ne pas trouver » $(8,17)$, «lot» $(2,20-21 ; 5,17-18 ; 9,9)$.

Le mot $k l$, "tout", qui précède le mot ' $m l$, est l'un des mots préférés de Qohélet, puisqu'il est employé 90 fois, dont dix autres fois en lien avec le mot ' $m l(1,3 ; 2,10.18 .19 .20 .22 ; 3,13 ; 4,4.8 ; 5,17)$. L'affirmation du v. 7 concerne donc tous les types de travail. En outre, elle concerne tout le monde, puisqu'il s'agit du labeur de l'être humain. En effet, le mot 'dm, précédé de l'article $h$, a ici un sens universel (Schoors 2004, 48).

Tout le labeur de l'être humain est littéralement "pour sa bouche» $\left(l_{p y h w}\right.$. Contrairement à son évocation dans les autres passages du livre, la bouche ne désigne pas ici l'organe de la parole (Qo 5,1.5; 8,2; 10,12.13 $[2 \mathrm{x}])$. Elle est plutôt l'organe du manger et du boire. Par ailleurs, en Qo 6,7 , la bouche peut également être synonyme de faim ou d'appétit, comme en Pr 16,26: «La faim/l'appétit ( $n p$ š) du travailleur (' $m l)$ travaille (' $m l h)$ pour lui, oui/car $(k y)$ ce qui le presse, c'est sa bouche/sa faim/son appétit $(p h) »$. Selon ce proverbe, qui a plusieurs termes en commun avec le v. 7, c'est la faim ou l'appétit qui pousse l'être humain (essentiellement un travailleur!) à travailler. Au v. 7a, le propos est semblable: l'être humain travaille pour satisfaire sa bouche, c'est-à-dire sa faim ou son appétit.

Toutefois, cette lecture est partielle et incomplète. En effet, les exégètes n'ont pas remarqué que la bouche peut aussi être l'organe du goût et donc de la délectation et de la jouissance (Jb 20,12; Ct 1,2; Si 40,30 et 49,1 du texte grec). C'est d'ailleurs ce que confirme le livre de Qo, car la jouissance et le bonheur sont décrits à l'aide des verbes manger et boire (2,24-25; $3,12-13 ; 8,15$ ). À l'instar de maints autres textes, le v. 7 souligne donc le lien intime qui unit labeur (' $\mathrm{ml}$ ) et bonheur (voir Qo 2,$24 ; 3,12-13 ; 5,18$; 8,$15 ; 9,9$ ). Autrement dit, le v. $7 \mathrm{a}$ a une double signification: travailler pour sa bouche, c'est non seulement travailler pour sa survie, mais aussi pour la jouissance que la bouche procure, donc pour le bonheur. 
Au v. 7b, Qohélet apporte une nuance importante à cette affirmation du v. 7a: wgm hnpš l' tml'. La traduction de l'expression $w g m$ ne fait pas l'unanimité. Certains omettent simplement de la traduire (L'Hour, Borel et Roubaud 2001, 1649). D'autres la rendent par la simple conjonction de coordination "et»(Glasser 1970, 91; Michaud 1987, 169). Faessler semble d'avis que la tournure $w g m$ dénote une addition, puisqu'il la traduit par "aussi» $(2013,131)$. À mon avis, le $w^{e}$ a ici un sens adversatif et le $g m$ qui suit vise à rendre ce sens adversatif encore plus explicite, d'où la traduction suivante: "mais pourtant». Bien entendu, ce n'est pas le seul passage où le mot $\mathrm{gm}$ peut avoir un sens adversatif (voir, par exemple, 2,$14 ; 4,8.16 ; 10,3$; etc.). Dans son analyse de Qo 2,14, Gordis affirme que cet emploi du mot gm avec un sens adversatif est même une caractéristique du style de Qohélet (1971, 222).

La tournure $w g m$ met l'accent sur le mot suivant, $n p \check{s}$, lequel est précédé de l'article $h$ qui est habituellement rendu par «le» ou «la» (Fox, 1999, 244; Schwienhorst-Schönberger 2004, 354; Fredericks 2010, 145). Toutefois, certains le rendent par «son" ou «sa» (Lauha, 1978, 107; Michel 1989, 147; Luzzatto 2011, 52). En effet, l'article $h$ équivaut parfois au pronom possessif (Joüon 1982, $422=137 \mathrm{f}$ ). Cette interprétation est d'autant plus vraisemblable que le mot $n p$ š correspond ici au mot $p h$ du v. $7 \mathrm{a}$, qui a le suffixe possessif. Or, ce suffixe peut très bien s'appliquer aux deux mots, comme c'est probablement aussi le cas en Qo 7,1 entre les mots hmwt et hwldw (Lauha 1978, 116).

Quoi qu'il en soit, le plus important est de savoir comment traduire le mot $n p$ š. La traduction par "âme» défendue par quelques exégètes (Schwienhorst-Schönberger 2004, 354; Bartholomew 2009, 233 et Wahl 2011, 62) ne respecte guère l'anthropologie moniste du livre de Qo et s'apparente davantage à une vision dualiste qui caractérise notamment l'anthropologie platonicienne. En effet, Qohélet n'emploie jamais le mot $n p s ̌$ dans un sens abstrait et désincarné. La traduction par "souffle" (De Luca 2007, 48) n'est guère plus juste, car ce mot, au sens anthropologique du terme, rend plutôt le terme rwh (Qo 3,19.21; 8,8 [2x]; 11,5; 12,7; pour le sens psychologique, voir Qo 7,8 [2x].9 et 10,4). À l'opposé, Luzzatto traduit le mot $n p$ š par «corps» $(2011,52)$. Il a certes raison d'utiliser un terme concret pour rendre le mot $n p$ š. Cependant, dans le livre de Qo, c'est plutôt le mot bśr, en tant qu'il désigne la personne tout entière, sans aucune forme de dualisme, qui doit être traduit par "corps» (Qo 2,3; 4,$5 ; 5,5 ; 11,10$ et 12,12 ). Plus précis, d'aucuns rendent $n p$ š par «gorge " (Ellermeier 1963, 20), "panse» (Steinmann 1955, 78) ou "estomac» 
(Michaud 1987, 169). Du point de vue philologique, ces traductions sont justifiées (voir, par exemple, Jr 31,14; Pr 3,22; 13,4.25; 16,24; 19,15; $25,25 ; 27,7)$. En outre, le parallèle avec le mot bouche au v. $7 \mathrm{a}$ indique bien que la traduction par gorge, gosier ou estomac est appropriée. Par ailleurs, à l'instar du mot $p h$ au v. $7 \mathrm{a}$, le mot $n p$ š peut aussi faire référence à l'appétit ou la faim (Pr 16,26;23,2; Dt 23,23; Os 9,4). C'est pourquoi quelques commentateurs traduisent $n p$ š par «faim» (Lauha 1978, 107; 116; Scippa 2010, 156), "appétit» (Fox 1999, 244) ou «besoin» (Scott 1965 , 231). Lu ainsi, le v. $7 b$ souligne que la gorge ou l'estomac n'est jamais rempli une fois pour toutes. À chaque jour l'appétit revient, la faim se fait sentir. C'est en quelque sorte la loi du «métro-boulot-dodo».

Aussi juste soit-elle, cette lecture est partielle. C'est par exemple ce qu'ont bien vu quelques exégètes qui, estimant que le v. $7 \mathrm{~b}$ ne saurait évoquer le seul besoin permanent d'assurer sa survie, préfèrent rendre le mot par «être» (Faessler 2013, 131), «personne» ou «moi» (Farmer 1991, 172) ou encore "désir» (Eaton 1983, 106). Ces traductions sont également justifiées du point de vue philologique et sémantique. Par exemple, en Jr 22,27 et 44,14, le mot $n p$ š exprime bien le désir de revenir au pays. Le mot a aussi très souvent le sens de «être" (Gn 2,7; 12,5; Lv 7,20; etc.). Enfin, dans le psautier, le mot est souvent l'équivalent du pronom personnel «moi» (Ps 103,1.2.22; 104,1.35; etc.).

À mon avis, la traduction par «être-de-désir» a l'avantage de souligner que le mot $n p s ̌$ désigne à la fois la faim, l'appétit, le désir et l'individu, le moi, l'être concret. Cette traduction a également l'avantage de bien rendre compte du sens du mot $n p s ̌$ non seulement dans les trois autres versets du chapitre 6 (Qo 6,2.3.9), mais aussi dans les trois autres passages du livre (Qo 2,$24 ; 4,8 ; 7,28$ ). En outre, cette traduction du mot $n p$ š par «être-dedésir» respecte le double sens du v. $7 \mathrm{~b}$. En effet, travailler pour se nourrir ne saurait combler l'être humain, car celui-ci a certes des besoins matériels, mais il est avant tout défini comme un être-de-désir. Par ailleurs, sachant que travailler pour sa bouche, c'est aussi travailler pour son bonheur, il n'est pas étonnant que Qohélet utilise le mot $n p s ̌$ pour affirmer que c'est le bonheur qui n'arrive pas à satisfaire l'être humain qui est un être-dedésir. En effet, un examen du livre de Qo indique que le désir par excellence de l'être-de-désir est le désir de bonheur (țwb/țwbh: Qo 2,24; 4,8; 6,3 ), qui se caractérise par des réalités concrètes: le manger et le boire (Qo $2,24)$, la richesse, les ressources et la gloire $(6,2)$ et la rencontre d'une femme (Qo 7,28; voir 9,7-10). En somme, tout le labeur de l'être humain a beau avoir pour but de lui procurer du bonheur (Qo 6,7a), l'être-de-désir 
qu'il est le condamne à rester un être insatisfait (Qo 6,7b). Il n'y a donc de satisfaction que restreinte et de bonheur que limité.

Cette limite est ici soulignée à l'aide du verbe $m l$ ', « remplir ", précédé de la négation l'. Or, dans le livre de Qo, l'emploi de ce verbe en lien avec l'être humain témoigne d'une vision anthropologique qui souligne bien la finitude humaine et la démesure du désir. D’une part, seul le cœur $(l b)$ de l'être humain est rempli ( $m l^{\prime}$ ), mais il est rempli de mal (Qo 8,11 et 9,3), c'est-à-dire rempli du désir de faire le mal! En effet, dans ce contexte, le verbe remplir ( $m l$ ') signifie "être plein de désir» ou "être obsédé »; c'est d'ailleurs ce que confirme l'emploi du verbe $m l$ ' suivi du mot cœur $(l b)$ en Est 7,5; Si 4,18 et 10,13 (Ms A). D'autre part, à l'instar de la mer, l'oreille, qui est l'organe de l'écoute et de la compréhension (1 Sam 9,15; 22,17; Is 22,14; Jb 33,16; etc.), n'est jamais remplie ( $m l$ ': Qo 1,7-8). Autrement dit, le désir de l'oreille, qui est d'envergure cosmique, n'est jamais satisfait. En Qo 1,8, la frustration du désir humain, indiquée par deux autres négations (l'ykl, «ne pas pouvoir ", et l'śb', «ne pas être rassasié»), s'étend aussi à l'œil ('yn) et à la bouche (par le biais du mot $d b r$ ). En outre, en Qo, le verbe śb', "rassasié», n'apparaît qu'avec la négation et toujours pour exprimer le caractère insatiable du désir humain (Qo 4,8; 5,9 et 6,3). L'emploi de ce verbe pour exprimer la frustration du désir n'est pas anodin, car c'est le même verbe, précédé de la négation, qui est utilisé pour décrire le caractère insatiable de la mort ( $\operatorname{Pr} 27,20$; 30,15-16; Hab 2,5). Autrement dit, le désir insatiable de l'être humain est un désir mortifère.

Ce lien entre désir et mort me conduit à soumettre une troisième lecture du v. 7. Celle-ci n'est pas entièrement nouvelle, mais certains arguments pour la justifier le sont. Jusqu'à maintenant, j'ai supposé que les mots $p h$ et $n p s ̌$ font exclusivement référence à l'être humain du v. 7a. Or, tel n'est pas l'avis de quelques exégètes qui jugent que ces mots se rapportent plutôt à l'expression mqwm 'hd, "Lieu unique ", du v. 6c, lequel fait clairement référence non pas à la "Transcendance insondable qui donne l'être » (Faessler 2013, 131), mais au monde des morts - ce qu'indique clairement l'emploi de la même expression en Qo 3,20 - donc au shéol (Ackroyd 1966-1967, 84-85; Dahood 1968, 368 ; Lauha 1978, 116; Lohfink 1980, 47). À mon avis, ces exégètes ont tort de croire que ces mots se rattachent exclusivement au mot mqwm. Par contre, il est vrai que, d'un strict point de vue grammatical et syntaxique, ils peuvent aussi se rapporter au mot mqwm du v. 6c. Du point de vue structurel, j'ai montré qu'il y a une continuité entre Qo 6,3-6 et 6,7-9. Par conséquent, le v. 7 est suffisamment ambigu pour qu'il puisse aussi se lire comme suit: tout le labeur 
de l'être humain est pour la bouche du Lieu unique, c'est-à-dire pour l'appétit destructeur de la mort, mais celui-ci n'est jamais satisfait. En effet, il est bien connu que le Shéol a une bouche $(p h)$ et une gorge $(n p s ̌)-$ précisément les deux mots employés en Qo 6,7! — pour saisir les vivants (Is 5,14 ; Hab 2,5). C'est encore à la bouche $(p h)$ du Shéol que les ossements sont dispersés (Ps 141,7). Être englouti vivant dans la bouche $(p h)$ de la Terre équivaut à descendre au shéol ( $\mathrm{Nb} \mathrm{16,30;} \mathrm{voir} \mathrm{aussi} \mathrm{Dt} \mathrm{11,6).} \mathrm{Le}$ Shéol avale les vivants $(\operatorname{Pr} 1,12)$ et Jonas se retrouve même dans son ventre (Jon 2,3). Enfin, comme au v. 7b, le Shéol, la Mort et l'Abîme, trois noms qui personnifient la même réalité, sont présentés comme des êtres insatiables (Pr 27,20; 30,15-16; Hab 2,5).

Ainsi, après avoir déclaré le non rassasiement de l'être-de-désir, malgré une progéniture nombreuse et une longue vie (Qo 6,3-6), Qohélet ajoute qu'il en va de même de la Mort (Lieu unique): elle aussi est insatiable (Qo $6,7 \mathrm{~b}$ ). En outre, ce n'est pas que l'être-de-désir qui est un être-vers-la-mort (Qo 6,3-6), mais c'est aussi tout son labeur (' $m l$ ) qui est destiné à la bouche du Lieu unique (Qo 6,7a), c'est-à-dire à l'anéantissement mortifère. Cette interprétation ne contredit aucunement le reste du livre de Qo. Au contraire, car ce qui reste à l'être humain de son labeur (' $m l)$, c'est rien d'autre que le non-être (Qo 2,22), le rien (Qo 5,14c). Enfin, à sa manière, Qo 1,9 confirme cette lecture, car ce passage souligne avec force que l'être et le faire ont aussi la même destinée (Qo 1,9).

En résumé, l'échec signalé en Qo 6,7 est triple. La nourriture ne peut combler l'être humain, d'une part, parce que la faim se fait sentir à nouveau chaque jour et, d'autre part, parce que l'être humain n'a pas que des besoins; il est fondamentalement un être-de-désir. En outre, de tous les désirs, celui du bonheur est le principal. Or, aucun bonheur n'arrive à combler l'être-de-désir. Enfin, comme c'est souvent le cas dans le livre de Qo, la mort se trouve à l'horizon de sa réflexion: tout le labeur de l'être humain, c'est-à-dire l'existence humaine elle-même puisque le labeur est perpétuel, est destiné à disparaître dans la bouche insatiable de la Mort.

\subsection{Verset 8}

Le v. 8 est introduit par la conjonction $k y$ que de nombreux exégètes omettent de traduire (Rose 1999, 319; Brandscheidt 1999, 188; Scippa 2010, 156; Wahl 2011, 62; etc.). D'aucuns donnent à cette conjonction un sens explicatif et la traduisent par «car» (Glasser 1970, 91; Fox 1999, 244; Pinçon 2011, 101), tandis que d'autres préfèrent lui donner un sens 
emphatique et la traduisent par «oui» (Schwienhorst-Schönberger 2004, 354; Faessler 2013, 131) ou "vraiment» (Lauha 1978, 107; Fredericks 2010, 145). Dans le premier cas, le v. 8 vient justifier le v. 7 , tandis que dans le second cas, il vient le renforcer. Dans les deux cas, le v. 8 est donc lié au v. 7.

Au thème du désir insatisfait fait donc suite une question $(m b)$ sur le profit-avantage (ywtr). Qohélet aime bien poser des questions. Il y a en effet 37 interrogations dans le livre: treize emplois de $m b(1,3 ; 2,2.12 .22$; 3,$9 ; 5,10.15 ; 6,8[2 \mathrm{x}] .11 ; 7,10 ; 8,4 ; 11,5$ [ou exclamatif]), quatre emplois de $\operatorname{lmb}(2,15 ; 5,5 ; 7,16-17)$, deux emplois de ' $y(2,16 ; 4,11)$, quinze emplois de $m y(2,19.25[2 \mathrm{x}] ; 3,21.22 ; 6,12$ [2x]; 7,13.24; 8,1 [2x].4 [ou exclamatif]. $7 ; 9,14 ; 10,14)$, un emploi de $\operatorname{lm} y(4,8)$ et deux emplois de $h$ $(3,21)$.

Les exégètes ont relevé depuis longtemps que la racine verbale $y t r$, qui signifie «rester ", revêt un sens commercial et désigne le surplus, le bénéfice ou le profit réalisé lors d'une transaction. Ce sens commercial est encore présent en Qo 5,8. Bien entendu, le mot a ici une signification anthropologique qui dépasse le sens strictement commercial. La question du profitavantage (ywtr) fait écho à Qo 1,3; 3,9 et 5,15 (dans les trois cas avec le mot ytrwn). Toutefois, ici, la question ne porte pas sur le profit du labeur de l'être humain en général, car celle-ci a déjà trouvé sa réponse au v. 7. La question porte plutôt sur l'avantage du sage sur l'insensé.

La racine $h \mathrm{~km}$ est commune dans le livre de Qo, car elle revient 53 fois, dont quatre fois comme verbe $(2,15.19 ; 7,16.23), 28$ fois comme substantif $(1,13.16$ [2x]. 17.18; 2,3.9.12.13.21.26; 7,10.11.12 [2x].19.23. $25 ; 8,1.16 ; 9,10.13 .15 .16[2 \mathrm{x}] .18 ; 10,1.10)$ et, comme ici, 21 fois sous forme d'adjectif, tantôt au singulier $(2,14.16[2 \mathrm{x}] .19 ; 4,13 ; 6,8 ; 7,5.7 .19$; $8,1.5 .17 ; 9,15 ; 10,2.12 ; 12,9)$, tantôt au pluriel $(7,4 ; 9,1.11 .17 ; 12,11)$. Dans le livre de Qo, le sage semble être de prime abord un personnage valorisé: il incarne celui qui sait $(2,14)$, qui médite sur la mort $(7,4)$, qui critique $(7,5)$, qui est rendu fort par la sagesse $(7,19)$, qui sait interpréter une parole ou un évènement $(8,1)$, qui sait qu'il y a un temps et un jugement $(8,5)$, qui a le cœur à sa droite $(10,2)$, qui parle avec grâce, en suscitant la faveur $(10,12)$ et qui peut libérer une ville $(9,15)$. En dernier lieu, le sage, c'est Qohélet lui-même (12,9).

Le mot $k^{e}$ sill est moins fréquent que le mot hākām. Il n'apparaît que dans les livres de sagesse ( $\mathrm{Pr}, \mathrm{Jb}$ et $\mathrm{Si}$ ), quelques psaumes (Ps 49,11.14; 78,$7 ; 92,7$ et 94,8) et dans le livre de Qo, où il revient 18 fois, tantôt au singulier $(2,14.15 .16[2 \mathrm{x}] ; 4,5.13 ; 5,2 ; 6,8 ; 7,6 ; 10,2.12)$, tantôt au plu- 
riel $(4,17 ; 5,3 ; 7,4.5 .9 ; 9,17$ et 10,15$)$. Quant au mot kesel, il n'apparaît qu'une seule fois en Qo 7,25. Le mot dérive probablement de la racine $k s l$, "être gras, lourd, épais", d'où le mot kāsel au sens de "lombes" ou "flanc» en Jb 15,27. La racine évoque donc celui qui est lourdaud, grossier, maladroit, d'où l'insensé, le stupide ou le fou. Un examen rapide des emplois de ce mot dans le livre de Qo indique qu'il est un personnage difficile à cerner avec exactitude. En plus d'apparaître comme l'opposé du sage, le $k^{e}$ sîl est un ignorant et un incompétent (Qo 2,14²;10,15b), un paresseux (Qo 4,5a $\mathrm{a}^{3}$ ) et un être qui s'autodétruit (Qo 4,5 $\mathrm{b}^{4} ; 10,12^{5}$ ). Le $k^{e}$ sil est également celui qui est épuisé par le travail (Qo 10,15a), qui ne sait plus se laisser conseiller (Qo 4,13), qui s'irrite (Qo 7,9), qui est attaché aux réjouissances de la vie (Qo 7,4; voir le texte grec de Sir 27,13) et qui s'imagine être le seul sage (Qo 10,3). Dans le domaine cultuel, il apparaît comme celui qui ne sait pas qu'il fait le mal (Qo 4,17), qui parle trop (Qo 5,2; voir $\operatorname{Pr} 18,2.6 .7$; 29,11; le texte hébreu de Si 20,6 et le texte grec de Si 20,7) et qui n'accomplit pas son voeu (Qo 5,3).

En résumé, le sage semble posséder un savoir intellectuel $(2,14 ; 7,4.5$; $8,1.5 ; 10,2.12)$ et un savoir pratique $(7,19 ; 9,15)$, tandis que l'insensé est un être qui ne se comporte pas comme il se doit et qui n'a ni sagesse, ni intelligence, aussi bien du point de vue pratique qu'intellectuel. Reste à savoir quelle est la réponse implicite à la question de l'avantage du sage sur l'insensé ? D'aucuns estiment qu'elle est positive (Fredericks 2010, 156). Déjà Aalders, à la suite de Levy et Thilo, estimait que le v. 8a était une véritable question, la réponse se trouvant au v. $8 \mathrm{~b}$ qui s'ouvre avec le mot mh compris comme un pronom relatif (Aalders cité dans Eaton 1983, 107). Fox est d'avis que l'avantage du sage sur l'insensé est relatif: le v. $8 \mathrm{a}$ ne nie pas catégoriquement l'avantage de la sagesse, mais seulement ce qui concerne la possibilité de parvenir à la satisfaction (Fox 1999, 245). À mon avis, comme c'est très souvent le cas en Qo, la question est rhétorique et la réponse ne peut être que négative (voir notamment Qo 1,3 et la réponse négative explicitement donnée en 2,11; Qo 3,9 où le «pour-tout » de 3,1-8

2. Les ténèbres symbolisent souvent l'ignorance ( $\mathrm{Jb} 12,24-25 ; 37,19 ; 38,2$ ). Inversement, les yeux et la lumière symbolisent la connaissance $(\mathrm{Si} 3,25)$.

3. L'expression «se croiser les mains » sert à décrire le paresseux en $\operatorname{Pr}$ 6,10 et 24,33.

4. L'acte de «dévorer sa propre chair» est un acte d'auto-extermination totale (voir aussi Is 49,26).

5. Littéralement, Qo 10,12b se traduit comme suit: "mais les lèvres d'un insensé l'avalent ", c'est-à-dire l'insensé se ruine par ses propres paroles. Le verbe $b l$ ', «avaler », " engloutir », "dévorer », rappelle le verbe ' $k l$, «manger» de Qo 4,5b. 
est un «pour-rien» et Qo 5,15 qui inclut la réponse dans la question: "pour du vent»!). C'est aussi ce qu'indique un examen des sept autres passages où le sage apparaît en compagnie de l'insensé $(2,14.15 .16 ; 4,13$; $7,4.5$; 10,2.12). C'est très clair en Qo $2,14-15$ qui rappelle que la mort annule toute forme de distinction entre le sage et l'insensé. Le sage comme l'insensé connaissent non seulement une mort identique, mais tous deux meurent en quelque sorte une deuxième fois, car ils finissent également par être oubliés (Qo 2,16; voir aussi 9,15). Bien qu'ils soient ambigus, les textes de Qo 4,13-16; 7,5-7; 10,2-3 et 10,12-15 relativisent, eux aussi et non sans ironie, la supériorité du sage sur l'insensé (Lavoie 2008, 15-39; 2014a, 1-26; 2014b, 59-84).

Le parallélisme entre les deux stiques du v. 8 indique que le mot $m b$ au v. $8 \mathrm{~b}$ introduit bel et bien une nouvelle question. Cette question a toutefois été traduite et comprise de différentes manières. Par exemple, Wahl croit que Qohélet compare le sage et le pauvre ('ny): "Qu'a de plus le sage par rapport au sot; par rapport au pauvre qui sait se conduire dans la vie?» (2011, 62). Cette traduction est erronée, car elle suppose que le mot $m b$ du v. $8 \mathrm{~b}$ a la même signification que le mot myn du v. $8 \mathrm{a}$. Il est vrai que, dans le livre de Qo, le mot $m b$ n'a pas toujours le sens interrogatif de l'hébreu classique. Toutefois, le seul autre sens qu'il a, c'est celui d'un pronom indéfini $(1,9[2 \mathrm{x}] ; 3,15.22 ; 6,10.12[2 \mathrm{x}] ; 7,24 ; 8,7 ; 10,14$ et 11,2), comme en araméen (Esd 6,8; 7,18; Dn 2,28.29.45). D'autres sont plutôt d'avis que le v. $8 \mathrm{~b}$ compare le pauvre ('ny) à celui qui sait $\left(y w d^{\prime}\right)$, mais c'est au prix d'une paraphrase injustifiée (voir Wright cité dans Eaton 1983, 107). Selon Faessler, la question du v. 8 b porte sur le savoir du pauvre ('ny): quel savoir pour l'humble ('ny) $(2013,131)$. Pourtant, force est de constater que, littéralement, l'interrogation du v. $8 \mathrm{~b}$ se lit plutôt comme suit: quoi pour le pauvre sachant...? Autrement dit: qu'a le pauvre qui sait...? Sachant que Qohélet a un style d'écriture elliptique, il est fort possible que le mot ywtr du v. 8a puisse être sous-entendu; par conséquent, il peut être ajouté entre parenthèses dans la traduction du v. 8b: quel (avantage) a le pauvre qui sait...? En effet, la construction du v. 8b est semblable à celle du v. $8 \mathrm{a}$ : dans les deux cas, la phrase commence par le pronom interrogatif $m b$ et le substantif qui suit est précédé d'un $l$, "pour». Ce type d'ellipse est bien connu. On le retrouve, par exemple, en Qo 10,13 où le mot "paroles" du v. 13a est sous-entendu au v. 13b.

Si le mot ' $n y$ ne pose guère de problème de traduction - en effet, de nombreux exégètes le traduisent par "pauvre" (Fox 1999, 244 ; Bartholomew 2009, 233; Scippa 2010, 156; Pinçon 2011, 102) —, il n'en 
va pas de même de la suite de la phrase. En effet, celle-ci peut être traduite de diverses façons: sachant marcher/aller/se conduire devant/contre les vivants/la vie. Le verbe $h l k$ étant à l'infinitif construit, la préposition $l$ qui le précède a ici un sens très faible, voire nul (Joüon 1982, 111-112 = 49f). Au plan syntaxique, la même construction (verbe $y w d^{`}$ au qal participe suivi de la préposition $l$ et d'un verbe à l'infinitif construit) apparaît en Qo 4,17 . Toutefois, on ne saurait fonder la signification du v. $8 \mathrm{~b}$ sur ce passage, car son interprétation est tout aussi controversée. Le verbe $y d^{\text {', }}$ "connaître", peut désigner un savoir faire, une habileté (voir Qo 8,1; 10,15; etc.) ou une connaissance plutôt intellectuelle (voir Qo 2,14.19; etc.). Par exemple, Seow défend la première interprétation $(1997,214)$, tandis que Faessler défend plutôt la seconde $(2103,132)$.

Quant au verbe $h l k$, il a été compris de différentes façons. Par exemple, à partir de son emploi en Qo 1,4, Perry est d'avis que ce verbe signifie aller de l'avant selon le désir ou le mouvement naturel. Il en déduit qu'il convient de traduire le v. $8 \mathrm{~b}$ comme suit: qui sait comment prendre la vie et jouir d'elle selon ses propres conditions? (1993, 108 et 116). Il est vrai que, dans le livre de Qo, le verbe hlk a de nombreuses significations. Toutefois, Qo 1,4 ne confirme aucunement cette interprétation trop psychologisante. Dans une tout autre perspective, Weeks est d'avis que le verbe $h l k$ a ici le sens de «mourir». Autrement dit, selon lui, la question du v. 8 b porte sur l'avantage du sage qui sait qu'il va quitter cette vie (2012, 98 et 115). Il est vrai, comme on va le voir au v. 9, que le verbe hlk a souvent le sens de mourir. Toutefois, un tel sens ne semble pas s'appliquer au v. 8b, car la connaissance que la mort est l'unique destin des êtres humains n'est aucunement un privilège du pauvre (Qo 2,15 ; 3,18-19; 5,14; 6,3-6; etc.).

Bien entendu, le verbe $h l k$ peut être compris dans son sens premier, soit celui de "marcher ", d' "aller» (voir Qo 1,6 [2x].7 [3x]; etc.). Ce sens est adopté par de nombreux exégètes (Rose 1999, 319; Faessler 2013, 131). Par contre, quelques commentateurs traduisent plutôt le verbe $h l k$ par «se conduire» (Michaud 1987, 169; Pinçon 2011, 102). À mon avis, il ne faut pas choisir entre l'un ou l'autre de ces deux sens. Il convient plutôt de reconnaître que le verbe $h l k$ au v. $8 \mathrm{~b}$ a ce double sens, comme en Qo 4,17; 7,2 et 10,3 (voir aussi, par exemple, 1 R 8,61 et $\operatorname{Pr} 10,9 ; 19,1$ ).

L'expression finale $n g d$ hhyym n'apparaît nulle part dans le reste de la Bible. Les deux mots formant cette expression sont également ambigus du point de vue sémantique. Le mot $n g d$ peut vouloir dire "devant» ou «contre ». Par exemple, Pinçon adopte la première traduction (2011, 102), tandis que Zimmerli opte pour la seconde $(1980,192)$. Le seul autre 
emploi du mot $n g d$ dans le livre de Qo se trouve en 4,12. Or, dans ce passage, le mot peut aussi avoir les deux significations. Quant au mot hyym, au pluriel, il peut faire référence aussi bien à la "vie» qu'aux "vivants". Le mot apparaît treize autres fois en Qo, tantôt au sens de "vie» $(2,3.17$; $3,12 ; 5,17.19 ; 6,12[2 \mathrm{x}] ; 8,15 ; 9,3.9[2 \mathrm{x}])$, tantôt au sens de "vivants" $(10,19)$. Zimmerli entérine la première traduction $(1980,192)$, tandis que Schwienhorst-Schönberger endosse la seconde (2004, 354).

En somme, il n'est donc pas étonnant que le v. $8 \mathrm{~b}$ ait été compris de diverses manières. D'aucuns croient qu'il est question de savoir faire face à la vie et donc de savoir réfléchir au problème de la vie (Gordis 1971, 170; Steinmann 1955, 78; Murphy 1992, 54). D'autres sont d'avis qu'il s'agit de savoir comment avancer avec les gens, voire de savoir comment les guider (Fox 1999, 245) ou comment marcher à la tête de tous (Rose 1999, 319). Certains jugent plutôt qu'il est question de savoir comment se conduire devant ou parmi les vivants (Michaud 1987, 169; Bonora 1992, 103; Bartholomew 2009, 233; Fredericks 2010, 145 ; Pinçon 2011, 102) ou devant ou dans la vie (Barucq 1968, 110; Maillot 1987, 89). Toutes ces traductions sont justifiées du point de vue grammatical et syntaxique.

Malgré les nombreuses ambiguïtés de ce v. 8 b, le mot ' $n y$ est un des mots clés qui permet de bien comprendre le sens de la question de Qohélet. Ce mot dérive de la racine ' $n h$. Du point de vue étymologique, le problème est de savoir s'il y a deux, trois ou quatre racines ' $n h$ et à laquelle de ces racines se rattache le mot ' $n y$. Whitley est d'avis que le mot " $n y$ se rattache à la racine " $n h$ au sens de "répondre", voire de répondre efficacement ( $\mathrm{Jb} 9,14-15)$ ou intelligemment $(\mathrm{Si} 9,14)$. Puis, estimant que ce mot est en parallèle synonymique avec le terme h $\mathrm{km}$, il le traduit par "homme intelligent» $(1979,59)$. Cette traduction est invraisemblable, car les anciennes versions ont toutes comprises le mot au sens de pauvre: peness, indigent (Septante), ptōchō, mendiant (Symmaque), pauper, pauvre (Vulgate), mskn', nécessiteux - mot qui apparaît aussi en Qo 4,13 et 9,15 [2x], mais pour rendre l'équivalent hébreu $m s k n$, qui est à l'origine du terme mesquin! - (version syriaque). En outre, s'il est vrai que la construction du début du v. 8 b est semblable à celle du v. $8 \mathrm{a}$, rien n'indique qu'il faille comprendre les mots h $\mathrm{km}$ et ' $n y$ comme des synonymes. Il est vrai que les mots mskn et $h \mathrm{~km}$ sont en parallèle en 4,13 et 9,15, mais les mots 'ny et hkm, eux, ne sont jamais des synonymes dans le reste de la Bible. À mon avis, le mot 'ny dérive de la racine verbale " $n h$ II, au sens de "opprimer", dont le sens étymologique désigne l'action de se courber ou d'être courbé. Le mot 'ny désigne 
donc celui qui plie et fléchit, celui qui est incapable de résister, le courbé, l'humilié, le faible, l'opprimé (Lavoie 2004, 72).

Du point de vue sémantique, le mot apparaît 27 fois en parallèle avec le terme 'bywn, le mendiant (Ps 9,19; 12,6; 35,10b; 37,14; 40,18; 70,6; 72,4.12; 74,$21 ; 86,1 ; 109,16.22 ; 140,13$; etc.), cinq fois avec le $d l$, le faible (Jb 34,28; $\operatorname{Pr} 22,22$; Is 10,2; 26,6; Soph 3,12), trois fois avec le gr, l'immigré (Lv 19,10; 23,22; Za 7,10) et une fois avec le $r \check{s}$, l'indigent (Ps 82,3). Un examen des 77 emplois du mot ' $n y$ indique qu'il n'apparait au pluriel que 21 fois. Bien que le mot 'ny soit parfois employé pour désigner le peuple de Dieu (2 Sam 22,28; Ps 18,$28 ; 72,2 ; 74,19$; Is 3,15; 49,13), le peuple de l'avenir (Sop 3,12), Jérusalem (Is 51,21; 54,11) et Anatôt (Is 10,30), il caractérise généralement au moins quatre types de personnes: le pauvre, celui qui est faible et impuissant dans le domaine social et économique (Ex 22,24; Lv 19,10; 23,22; Dt 15,11; 24,12.14; etc.), l'opprimé, celui qui est malheureux (Ps 10,2.9.12; 18,28; 25,16; 109,22; etc.), l'humble, le modèle de la confiance en Dieu (Ps 22,25; 25,$16 ; 34,7 ; 40,18 ; 69,30 ; 70,6 ; 86,1 ; 102,1 ; 109,22$; etc.) et le roi messie (Za 9,9). Le mot 'ny peut donc être rendu par pauvre, opprimé ou humble. Dieu est du côté du 'ny et c'est lui qui assure son salut (Ps 12,6;14,6;18,28; 22,$25 ; 34,7 ; 35,10 ; 40,18 ; 68,11 ; 69,30 ; 70,6 ; 140,13$; etc.) et lui accorde sa faveur ou sa bonté $(\operatorname{Pr} 3,34)$. C'est également Dieu qui demande au roi (Ps 72 , 2.4) et aux dieux (Ps 82,3) de rétablir son droit.

La question rhétorique du v. 8b, qui suppose une réponse négative, indique que Qohélet refuse d'idéaliser la spiritualité de la pauvreté qui est déjà bien en vogue au retour de l'exil. C'est ce qu'avait déjà remarqué Schwienhorts-Schönberger qui est d'avis que Qohélet critique une fausse compréhension de la spiritualité du pauvre (2004, 356-357). Toutefois, sa traduction (qu'a le pauvre qui sait marcher parmi les vivants ?) et les rapprochements qu'il fait, d'une part, avec les Ps 56,14 et 116,8-9 et, d'autre part, avec le Ps 73,26-28, ne sont pas convaincants. Il est vrai que l'on trouve bel et bien le mot hyym dans les deux premiers psaumes, mais à l'intérieur d'une formule qui ne s'apparente aucunement à celle de Qo 6,8b. Il est donc injustifié de donner un sens eschatologique à Qo 6,8b à partir de ces deux psaumes. Il est également vrai qu'il y a un réel contraste entre la conception de la part $(h l q)$ et du bonheur $(t w b)$ de Qohélet (voir notamment 3,$22 ; 5,17-18 ; 9,9$ ) et celle de l'orant au Ps 73 , qui affirme que Dieu est sa part $(h l q)$ pour toujours (v. 26) et que son bonheur (twb) est d'être près de Dieu (v. 28). Toutefois, force est de constater que ce psaume ne fait aucune mention du pauvre ('ny) et que Qohélet, au v. 8, ne fait aucune mention de bonheur et de part. 
À mon avis, pour comprendre pleinement la critique de la spiritualité du pauvre, il faut respecter l'ambiguiité de la formulation du v. 8b. C'est ce qu'indiquent les trois traductions suivantes, qui sont également justifiées du point de vue grammatical, sémantique et syntaxique. Qu'a/quel avantage a le pauvre /l'humble qui sait marcher devant les vivants / qui sait marcher à la tête des vivants? Cette première traduction permet de comprendre que Qohélet conteste les textes de sa tradition, notamment ceux du psautier, qui présentent le 'ny comme le modèle à suivre. En outre, sachant que Za 9,9 présente le roi messie comme un ' $n y$ qui va apporter la libération (Za 9,10-16) et le bonheur (țb: Za 9,17), on peut aussi voir dans le v. 8 b une critique à peine voilée de ce messianisme de la pauvreté. Ce n'est d'ailleurs pas le seul passage du livre où Qohélet critique les espérances prophétiques, qu'elles soient messianiques (Qo 4,13-16) ou eschatologiques (voir surtout Qo 1,9-11) (Lavoie 2008, 15-39; Krüger 1996, 107-130). Qu'a/quel avantage a le pauvre l'bumble qui sait faire face à la vie/aux vivants? Cette deuxième traduction permet de saisir que Qohélet conteste aussi les textes du psautier qui présentent le 'ny comme le modèle du croyant qui sait faire face à l'adversité. Qu'a/quel avantage a le pauvre/l'humble qui sait se conduire devant les vivants/dans la vie? Enfin, cette troisième traduction permet de découvrir que Qohélet conteste les textes du psautier qui présentent le 'ny comme un modèle à suivre par la manière dont il se conduit, c'est-à-dire par l'entière confiance qu'il met en Dieu. En somme, Qohélet refuse de faire du 'ny un personnage exemplaire (modèle du guide à suivre, d'où la figure de l'humble messie) et un modèle du croyant qui, sachant faire face à l'adversité et sachant bien se conduire (en mettant son entière confiance en Dieu), est le bénéficiaire de l'intervention divine. Le pauvre restera toujours pauvre. Autrement dit, son savoir ne lui procure aucun avantage.

\subsection{Verset 9}

Dans la traduction de la JPS, le v. 9a est rendu comme une question: est ce que la fête des yeux est plus importante que la poursuite du désir? (Fox 2004, 41). Au contraire, Faessler traduit le v. 9a comme une affirmation: le bien c'est l'évidence des yeux plutôt que la poursuite de l'être (2013, 131). À mon avis, ces deux traductions sont des paraphrases qui ne rendent pas compte du sens et de la forme littéraire du texte. En effet, le v. 9 s'ouvre par un dit de préférence $(t w b+m[n])$, une forme littéraire qui revient fréquemment dans le livre de Qo $(4,3.6 .13 ; 5,4 ; 6,3.9 ; 7,1 \mathrm{a}$ 
[b].2a.3a. 5a. 8ab; 9,4.16.18). Le mot țwb, qui revient 52 fois dans le livre de Qo et avec différentes significations selon les contextes, exprime donc ici un «mieux». Or, le "mieux» n'est pas le «bien»; cette nuance est importante, car le «mieux» présente certes un avantage, mais celui-ci est forcément relatif.

L'expression qui suit, $m r^{\prime} h$ 'ynym, littéralement "vision des yeux », a donné lieu à diverses interprétations. D'aucuns estiment que l'expression est synonyme de simple vue et donc de contemplation sans jouissance (Asurmendi 2012, 94; Fredericks 2010, 145 et 147). Asurmendi ne présente aucun argument pour étayer son interprétation. Il présuppose, sans aucun examen, que l'œil est le simple organe de la vue. De son côté, Fredericks justifie son interprétation en invoquant un argument structurel: Qo 6,9 correspond à Qo 5,10 et doit donc avoir la même signification. Or, j'ai montré ci-dessus la faiblesse de son analyse structurelle. En outre, la formule employée en Qo 5,10 ( $r^{\prime} y t$ ' $y n y w$, «vue de ses yeux») n'est pas la même que celle de Qo 6,9 et n'a donc pas la même signification; en effet, le contexte du v. 10 indique clairement que la formule a une connotation négative et qu'elle désigne le simple spectacle pour les yeux (Laurent 2002, 58). D'autres sont plutôt d'avis que l'expression "vision des yeux" est l'équivalent d'être en vie (Bartholomew 2009, 237; Enns 2011, 76). Ils ne citent toutefois aucun texte à l'appui de leur interprétation. Il est vrai que «voir le soleil » équivaut à vivre (Qo 6,5; 7,11 et 11,7), mais force est de constater que cette expression est différente de celle du v. 9a. C'est pourquoi cette interprétation me semble indéfendable. De son côté, Pinker croit que l'expression $m r$ 'h 'ynym ne désigne que l'apparence (Pinker 2009, 220). Pour justifier son interprétation, il affirme que le mot $m r^{\prime} h$, dans le reste de la Bible, fait toujours référence à la vue, l'apparence, la vision. En outre, il estime que l'expression quasi identique en Qo 11,9d fait référence à une vision superficielle et éphémère. Pour justifier cette interprétation, il compare le stique parallèle, soit Qo 11,9c, à Is 57,17. À mon avis, cette comparaison est boiteuse, car le texte et le contexte d'Is 57,7 sont différents, comme en témoigne, par exemple, la présence du mot šôbāb qui est absent du texte de Qo 11,9: «il est allé rebelle (šôbāb) dans le chemin de son cœur». Quant à l'emploi du mot mr'h dans le reste de la Bible, il ne désigne pas qu'une apparence. Au contraire, en dehors du livre de Qo, dans quatre des cinq autres passages où apparaît l'expression $m r$ ' $h$ 'nym, celle-ci désigne bel et bien une visions réelle (voir Lv 13,12; Dt 28,34.67; Ez 23,16; la seule exception est Is 11,3 où l'expression peut désigner une apparence). 
Quoi qu'il en soit, aucun de ces cinq passages ne permet vraiment d'éclairer le dit de préférence du v. 9a, car les contextes sont trop différents. Par ailleurs, la même expression apparaît en Qo 11,9d. Or, dans ce dernier passage, le contexte indique que les expressions "chemin de ton cœur» et "visions de tes yeux» (wbmr'y 'ynyk) ont indéniablement un sens positif. En effet, Seow a bien montré qu'elles expriment de manière concrète la réalisation de la joie de vivre à laquelle Qohélet exhorte le jeune homme (1997, 349-350). Autrement dit, les visions des yeux n'impliquent pas qu'une banale perception des choses par le regard; elles signifient une réelle expérimentation de la joie (voir aussi les verbes śmh et $y t ̣ b$ en 11,9ab). Cette interprétation est d'ailleurs confirmée par un texte talmudique (Yoma 74b) dans lequel l'expression $m r^{\prime} h$-'ynym b’šb signifie le plaisir de regarder une femme (Whitley 1979, 60).

Un examen du verbe $r$ ' $h$ dans le livre de Qo confirme également cette interprétation. En effet, ce verbe a souvent le sens précis de goûter et d'apprécier le bonheur $(2,1.24 ; 3,13 ; 5,17 ; 6,6 ; 9,9)$. Par ailleurs, dans le livre de Qo, l'œil est aussi l'organe de la connaissance $(2,14)$, du sommeil $(8,16)$, des émotions et du désir $(1,8 ; 2,10 ; 4,8)$ et plus précisément de la jouissance $(4,8 ; 11,7.9)$.

En somme, en Qo 6,9a, l'expression «vision des yeux» connote donc l'idée d'expérimenter concrètement le plaisir et la joie. Le dit de préférence peut donc être interprété comme suit: mieux vaut la jouissance effective ou mieux vaut expérimenter immédiatement le bonheur.

L'expression de la deuxième partie du dit de préférence, $m b l k-n p s ̌$, a donné lieu, elle aussi, à diverses interprétations. Selon Pinker, le mot $n p s ̌$ désigne ici la gorge, tandis que le verbe $h l k$ signifie simplement "aller» $(2009,221)$; il traduit donc le v. 9a comme suit: mieux vaut ce que voient les yeux que ce qui va dans la gorge; autrement dit, l'apparence est meilleure que le goût $(2009,224-225)$. Il est vrai que le mot $n p s ̌$ peut désigner la "gorge " et que le verbe hlk signifie littéralement «aller» ou «marcher». Toutefois, la tournure mblk est à l'infinitif construit (littéralement: que l'aller de) et le mot npš n'est pas précédé de la préposition $b$, comme le suppose la traduction de Pinker. En outre, ayant déjà montré au v. 8 que le verbe $h l k$ peut avoir plus d'un sens dans Qo, rien ne nous oblige à lui donner son sens littéral, ni même les sens qu'il a au v. 8. Ce type d'antanaklasis n'est pas rare en hébreu biblique et apparaît ailleurs en Qo. L'emploi du verbe māṣā' en Qo 7,23-29 et du verbe nûha en Qo 10,4ab en sont deux bons exemples (Lavoie 2013, 376). 
La traduction allemande (Einheitsübersetzung) que commente et endosse Lohfink rend l'expression mblk-npš par "gorge affamée» (1980, $48)$ et cette traduction est reprise par Stendebach $(2001,36)$. Cette paraphrase est injustifiée, car le verbe hlk n'a jamais ce sens dans le reste de la Bible. De nombreux exégètes estiment que le verbe $h l k$ est l'équivalent du verbe «errer» ou "vagabonder». Ceux qui défendent cette traduction n'en déduisent pas pour autant la même interprétation. Par exemple, Lange affirme que l'errance de la $n p s ̌$ décrit un processus d'activité de la sagesse (1991, 135), tandis que Michel est d'avis que l'errance de la npš est synonyme de "désir » : mieux vaut la vue (= jouir) des yeux que l'errance de la $n p \check{s}$ (= le désir) (Michel 1989, 150). Pinker rejette sans aucune discussion cette équivalence entre $h l k$ et "errer ", sans doute parce que les auteurs qu'il cite ne présentent aucun argument pour la justifier (2009, 220 et 225). En effet, rares sont les exégètes qui prennent le soin de justifier cette équivalence. Par exemple, Fox évoque l'emploi du verbe $h l k$ en $\operatorname{Pr} 14,7$. Cet argument est peu convaincant, car l'expression $l k m n g d$, littéralement «va de devant», a plutôt le sens de "fuir» ou de «s'éloigner»: "Fuis l'homme insensé» (Pr 14,7a). De son côté, sans aucune explication, Krüger cite simplement l'expression rhembasmos epithumias de Sg 4,12 (2004, 118). Le problème, c'est que le mot rhembasmos, "agitation", "inquiétude", est un hapax. Il est formé sur le verbe rhembazo, dont le sens fondamental est le même que celui des verbes rhembō et rhembomai: «tournoyer", "s'agiter tout autour» (Bailly 1919, 1715). Or, ces verbes n'apparaissent que deux fois dans la Septante. Le verbe rhembomai est employé en $\operatorname{Pr}$ 7,12 pour décrire l'agitation de la femme qui cherche à tendre une embuscade à un homme. Toutefois, il est impossible de tirer une conclusion de ce passage, car il est sans correspondant dans le texte hébreu. Quant au verbe $r$ hembō, il est employé en Is 23,16 sous la forme impérative: rhembeuson poleis, "fais le tour de la ville». Or, dans ce texte, il traduit le verbe $s b b$, "faire le tour", et non le verbe hlk, "aller", "marcher». Bref, cet appel au texte e Sg 4,12 n'est pas plus convaincant que l'appel à Pr 14,7. Enfin, la comparaison signalée par Di Fonzo $(1967,219)$ entre l'expression du v. 9 et la hormē tēs psuchēs de Marc Aurèle (Livre $3,16)$ n'est guère plus concluante. Il est vrai que le mot hormē peut désigner à la fois le départ pour une marche, le voyage et, chez les Stoïciens, l'impulsion, l'instinct (Bailly 1919, 1403). Toutefois, l'expression hormē tês psuchēs est trop tardive ( $\mathrm{II}^{\mathrm{e}}$ siècle de l'ère chrétienne!) pour que l'on puisse s'en servir afin de comprendre Qo 6,9. En outre, l'emploi du mot hormē dans la Septante ne confirme aucunement ce rapprochement. En 
effet, l'expression hormē tēs psuchēs est absente de la Septante et le mot hormē ne traduit jamais le verbe hlk (voir Nb 11,11.17; $\operatorname{Pr} 3,25 ; 21,1 ; \mathrm{Za}$ $7,12 ; \mathrm{Jr} 29,3 ; \mathrm{Ez} 3,14)$. En définitive, les arguments évoqués pour justifier la traduction du verbe $h l k$ par «errer» ne sont pas convaincants.

À mon avis, le verbe hlk peut avoir ici un double sens. Il peut d'abord avoir le sens de "poursuivre", comme au Ps 131,1: "Yhwh, mon cœur n'est pas orgueilleux et mes yeux ne se sont pas élevés, je n'ai pas poursuivi (blkty) des grandeurs et des merveilles qui me dépassent ». Ce passage, qui décrit ce que la confiance en Dieu n'est pas, est d'autant plus intéressant que le verset suivant décrit cette confiance en termes positifs: "Au contraire, mon être-de-désir ( $n p s ̌)$ est tranquille et silencieux, tel un [enfant] rassasié sur sa mère, tel un [enfant] rassasié, mon être-de-désir (npš) en moi. » (Ps 131,2). Ainsi, l'acte de poursuivre ( $h l k)$ est ici opposé à l'état de l'être-de-désir qui est rassasié. De la même façon, l'expression $m b l k-n p \check{s}$ en Qo 6,9 peut donc signifier "poursuivre un désir». Autrement dit, mieux vaut la jouissance concrète et immédiate que la poursuite de désir. Certes, aucun bonheur n'arrive à combler l'être-de-désir (Qo 6,7), mais le bonheur concret et immédiat n'en reste pas moins préférable à la poursuite de désir. Cette interprétation est conforme au reste du livre, car Qohélet souligne à maintes reprises le fait qu'il n'y a de bonheur ('yn twb) que dans des jouissances et des plaisirs quotidiens: le manger, le boire, le bonheur issu du labeur (Qo 2,24; 3,12-13.22; 8,15; voir aussi 5,17 et 9,7.9). En effet, sachant que l'être humain est incapable de déchiffrer le temps (Qo 3,11$12 ; 9,11-12)$, que la vie est éphémère et que la mort peut survenir à chaque instant (Qo 5,17; 8,15; 9,9-12), Qohélet soutient que l'être humain doit se contenter de jouir du moment présent, si Dieu l'y autorise (Qo 5,18; 6,1-2).

Par ailleurs, quelques exégètes donnent un autre sens à l'expression $m b l k-n p s ̌$. Sachant que le mot npš désigne aussi la «vie", Strobel estime que l'expression désigne l'aventure risquée de la vie (1967, 100). Pour justifier son interprétation, il cite deux textes où l'on trouve une expression similaire: hblkym bnpšwtm, "qui sont allés au péril de leur vie» (2 Sam $23,17)$ et $w y l k$ ' $l-n p s ̌ w$, «et il partit pour sauver sa vie» (1 $\mathrm{R} 19,3)$. Quelques exégètes croient plutôt que l'expression $m b l k-n p s ̌$ désigne plus précisément le "passage de la vie ", c'est-à-dire la mort (Loader 1979, 86; Whitley 1979, 60; Whybray 1989, 109; Bartholomew 233 et 238; Weeks 2012, 98). À mon avis, ils ont tort d'y voir cet unique sens, mais ils ont raison de défendre cette traduction, car il est vrai que le verbe hlk a souvent le sens de «mourir» dans le livre de Qo $(1,4 ; 3,20 ; 5,14.15 ; 6,4.6$; 
9,10 et 12,5). Ainsi, le dit de préférence du v. 9a est ambigu puisqu'il peut également avoir cet autre sens: mieux vaut la jouissance immédiate que la mort, et plus précisément la mort de l'être-de-désir. Compris ainsi, le dit de préférence du v. 9a vient donc nuancer Qo 6,3-6. S’il est vrai que la mort est préférable à une vie sans bonheur (Qo 6,3.6), la vie avec bonheur, elle, est préférable à la mort! C'est sans doute pourquoi le bonheur fera l'objet de recommandations (Qo 9,7-9; 11,9-12,1a).

En ce qui concerne le verdict final du v. 9 ( $g m$ zh hbl wr'wt rwh), il suscite deux grandes questions. À quoi se rapporte-t-il ? Comment doit-on le traduire? Les réponses des exégètes à la première question sont nombreuses. Perry affirme que ce verdict se rapporte aux v. 10-11 qui suivent (1993, 117). À mon avis, c'est impossible, car, du point de vue de la critique des formes, l'expression $g m z h h b l$ est habituellement utilisée comme formule conclusive (Qo 2,11.23.26; 4,8.16), sans qu'elle n'indique pour autant la fin d'une péricope (Qo 2,15.19.21; 4,4; 5,9; 7,6; 8,10.14). En outre, j'ai déjà signalé lors de ma critique structurelle que l'expression $r^{6} w t$ rwh clôt toujours une réflexion ou une péricope. Dans sa spéculation philosophique, qui a une fois de plus peu à voir avec le texte de Qo, Faessler affirme qu'il «est aisé de saisir» que le verdict «touche la prétention d'un savoir ontologique qui colmate ses propres limites en absolutisant son idéologie » $(2013,132)$ ! De manière plus précise, d'aucuns croient que le verdict se rapporte à la seule deuxième partie du v. 9a (Michel 1989, 358; Schwienhorst-Schönberger 2004, 358; Wahl 2011, 62), à l'ensemble du v. 9a (Murphy 1992, 54-55; Fox 1999, 246), aux v. 7a et 9a (Fredericks 2010, 156), aux v. 7-9a ou plus particulièrement au travail, à la sagesse et aux plaisirs (Miller 1998, 447), voire à l'ensemble de la section qui va de 5,7 à 6,9a (Enns 2011, 76) ou de 5,9 à 6,9a (Longman 1998, 175).

Force est de constater que les réponses sont nombreuses et plutôt variées! Doit-on rattacher le verdict à la seule deuxième partie du v. 9a? C'est improbable, car un tel jugement défavorable et emphatique sur la poursuite du désir ou sur la réalité de la mort va de soi et serait inutilement démesuré. L'emploi de la formule $g m z h$, "cela aussi », indique plutôt que le verdict s'applique tout autant à ce qui est positif qu'à ce qui est négatif. On peut dès lors rattacher ce verdict à l'ensemble du v. 9a. Faut-il également rattacher ce verdict aux v. 7-8 qui précèdent ? C'est fort possible, car j'ai bien montré dans ma critique structurelle que ces v. 7-9 forment une petite unité. C'est d'autant plus probable que l'on sait que l'expression $\mathrm{bbl}$ hblym en Qo 1,2 forme une inclusion avec Qo 12,8. Qo 1,2 n'ouvre donc le livre (Qo 1,2) que parce qu'il en donne d'avance la conclusion (Qo 
12,8). Cette inclusion montre ainsi que tout le développement intermédiaire constitue en quelque sorte un commentaire de cette unique sentence.

Reste à savoir comment traduire ce verdict, notamment le mot $h b l$, mot-clé par excellence de tout le livre. Bien entendu, cette seule question a déjà fait l'objet de nombreuses études qu'il n'est pas question de reprendre ici (Lavoie 2006, 219-249). Comme le verdict vise à relativiser ce qui précède, la traduction par absurdité (au sens de ce qui est à la fois inexplicable, déraisonnable, et incohérent) me semble adéquate.

Quant à l'expression $r^{6} w t r w h$, qui apparaît ici pour la dernière fois (voir Qo 2,11.17.26; 4,4.6), elle peut être rendue par "poursuite de vent/souffle» ou "pâturage de vent/souffle»(Lavoie 2007, 103-105). Dans cette expression, le mot rwh représente à la fois ce qui n'a aucune substance (voir Pr 11,29; 27,16; 30,4; etc.), ce qui échappe complètement au pouvoir des êtres humains (Qo 11,4-5) et ce qui n'appartient qu'au Créateur (Qo 3,21; 12,7). En définitive, l'expression $r^{\prime} w t$ rwh exprime à la fois un effort parfaitement stérile et insignifiant, un travail impossible et un désir de maîtrise de ce qui n'appartient qu'à Dieu.

\section{Pour ne pas conclure...}

Le désir est illimité. C'est clair pour Qohélet en 6,7.9a. Pour les psychanalystes, c'est même un truisme. L'être humain n'est donc jamais satisfait (Qo $1,7-8 ; 4,8 ; 5,9 ; 6,3.7)$. Pour les exégètes, l'insatisfaction se manifeste de manière particulière lorsqu'ils lisent les commentaires du livre de Qo! Mais aussi, sans doute - c'est du moins mon cas — lorsqu'ils se prêtent euxmêmes au jeu du commentaire! Pourtant, il faut savoir se limiter et donc savoir conclure, sans pour autant prétendre avoir dit le dernier mot.

Or, que conclure sinon que Qo 6,7-9 est un passage énigmatique qui pose des difficultés des points de vue textuel, structurel, syntaxique, sémantique et référentiel ? Du point de vue textuel, j'ai montré que le texte massorétique de 6,7-9 ne nécessite aucune correction, même si les anciennes versions témoignent de diverses leçons et traductions. Du point de vue structurel, j'ai relevé de nombreux indices qui montrent que ces trois versets, qui ne sont pas sans lien avec ce qui précède, forment une petite unité qui peut très bien se comprendre telle qu'elle se donne à lire maintenant. Il est donc inutile d'imaginer l'intervention d'un rédacteur quelconque ou de présupposer que Qohélet cite un ou des textes de la sagesse traditionnelle.

Du point de vue grammatical, sémantique, syntaxique et référentiel, j'ai bien montré que ces trois versets sont ambigus et qu'ils se prêtent à de 
nombreuses interprétations qui, loin de se contredire, se complètent très bien. En ce qui concerne le v. 7, j'ai relevé trois interprétations possibles, qui peuvent se résumer comme suit: tout le labeur de l'être humain est pour sa bouche, mais sa faim n'est jamais assouvie une fois pour toutes; tout le labeur de l'être humain est pour son bonheur, mais l'être-de-désir n'est jamais satisfait; tout le labeur de l'être humain est destiné à disparaître dans la gueule insatiable de la Mort. Le v. 8 a ne pose guère de difficulté: par le biais d'une interrogation rhétorique qui suppose une réponse négative, j'ai montré que Qohélet rappelle que le sage n'a pas de réel avantage sur l'insensé. La question du v. 8 b est également rhétorique, mais j'ai souligné qu'elle vise à faire une critique de la spiritualité de la pauvreté qui est déjà bien en vogue au retour de l'exil, notamment dans le psautier. En résumé, Qohélet refuse de faire du pauvre ou de l'humble un personnage exemplaire (modèle du guide à suivre, d'où la figure de l'humble roi messie) et un modèle du croyant qui, sachant faire face à l'adversité et sachant bien se conduire (en mettant son entière confiance en Dieu), est le bénéficiaire de l'intervention divine. Le pauvre restera toujours pauvre. Autrement dit, son savoir ne lui procure aucun avantage. Dans mon analyse du v. 9a, j'ai montré qu'il est tout aussi ambigu que les versets précédents, puisqu'il peut se lire de deux manières: mieux vaut une jouissance concrète et immédiate que la poursuite (sans fin!) du désir ou que la mort. Enfin, j'ai indiqué les principales raisons qui expliquent pourquoi le verdict final du v. 9b peut aussi bien faire référence au v. 9a qu'à l'ensemble des v. 7-9a.

Reste alors une question: ces ambiguités grammaticales, sémantiques, syntaxiques et référentielles sont-elles le produit d'une négligence littéraire ou sont-elles au service d'une stratégie rhétorique qui caractérise l'ensemble du livre? À mon avis, les ambiguïtés sont trop nombreuses dans le livre de Qo pour être considérées comme accidentelles. Elles sont plutôt au service d'une stratégie rhétorique qui vise à inciter le lecteur à éprouver différentes lectures, même si le risque est grand que celui-ci n'en retienne qu'une seule, celle qui va correspondre le mieux à sa propre sensibilité, sa propre expérience et, bien sûr, ses propres préjugés. Mais le risque mérite d'être pris, car la stratégie rhétorique de Qohélet ne vise-t-elle pas aussi à solliciter l'intelligence du lecteur et à alimenter son désir de savoir ? Or, le désir de savoir n'est-il pas, par excellence, le désir qui habite tout lecteur du livre de Qo? Qui plus est, ce désir de savoir ne se déploie-t-il pas que dans la différence, c'est-à-dire dans la reconnaissance de l'altérité du texte commenté et donc du caractère inachevé de tout commentaire? 


\section{Références}

Ackroyd, P. R. (1966-1967), "Two Hebrew Notes", Annual of the Swedish Teological Institute, 5, p. 84-86.

Alonso Diaz, J. (1967), En lucha con le Misterio. Le alma judia antes los premios y castigos y la vida ultraterrena, Santander, Editorial Sal Terrae.

Anaya Luengo, P. R. (2007), El Hombre, destinatario de los dones de Dios en el Qohélet, Salamanca, Publicaciones Universidad Pontificia, (Bibliotheca Salmanticensis 296).

Asurmendi, J. (2012), Du non-sens. L’Ecclésiaste, Paris, Cerf (Lectio Divina 249).

Bailly, A. (1919), Dictionnaire Grec-Français, Paris, Hachette.

Bartholomew, C. G. (2009), Ecclesiastes, Grand Rapids, Baker Academic.

BarucQ, A. (1968), Ecclésiaste, Qohéleth. Traduction et commentaire, Paris, Beauchesne (Verbum Salutis AT 3).

Biblia Sacra iuxta latinam Vulgatam versionem (1957), Romae, Typis Polyglottis Vaticanis.

Birnbaum E. et Schwienhorst-Schönberger, L. (2012), Das Buch Kohelet, Stuttgart, Verlag Katholisches Bibelwerk (Neuer Stuttgarter Kommentar Altes Testament 14/2).

Bonora, A. (1992), Il libro di Qoèlet, Roma, Città Nuova (Guide Spirituali all'Antico Testamento).

Botтéro, J. (1986), "L’Ecclésiaste et le problème du mal », Naissance de Dieu. La Bible et l'historien, Paris, Gallimard, p. 222-251.

BRANDSCHeIDT, R. (1999), Weltbegeisterung und Offenbarungsglaube. Literar-, form- und traditionsgeschichtliche Untersuchung zum Buch Kohelet, Trier, Paulinus (Trierer Theologische Studien 64).

Buzy, D. (1946), "L'Ecclésiaste", dans L. Pirot et A. Clamer, dir., La Sainte Bible, Vol. 6, Paris, Letouzey et Ané, p. 189-280.

Coppens, J. (1979), "La structure de l'Ecclésiaste», La sagesse de l'Ancien Testament, Gembloux, Duculot (Bibliotheca Ephemeridum Theologicarum Lovaniensium 51), p. 288-292.

Costaz, L. (2003), Grammaire syriaque, Beyrouth, Dar el-Machreq.

Dahood, M. (1968), «Hebrew-Ugaritic Lexicography VI », Biblica, 49, p. 355-369.

Delsman, W. C. (2000), Die Datierung des Buches Qoheleth. Eine sprachwissenschaftliche Analyse, Nijmegen, Nijmegen University Press. 
De Luca, E. (2007), Kohèlet/Ecclesiaste, Milano, Feltrinelli (I Classici Universale Economica Feltrinelli 2137).

Di Fonzo, L. (1967), Ecclesiaste, Torino-Roma, Marietti.

Eaton, M. A. (1983), Ecclesiastes. An Introduction and Commentary, InterVarsity Press (The Tyndale Old Testament Commentaries 16).

Ellermeier, F. (1963), «Die Entmachtung der Weisheit in Denken Qohelets. $\mathrm{Zu}$ Text und Auslegung von Qoh 6,7-9", Zeitschrift für Theologie und Kirche, 60, p. 1-20.

Enns, P. (2011), Ecclesiastes, Grand Rapids, Eerdmans (The Two Horizons Old Testament Commentary).

Faeesler, M. (2013), Qohélet philosophe. L'éphémère et la joie. Commentaire herméneutique de l'Ecclésiaste, Genève, Labor et Fides.

Farmer, K. A. (1991), Who Knows what is Good? A Commentary on the Books of Proverbs ans Ecclesiastes, Grand Rapids, Eerdmans (International Theological Commentary).

Field, F. (1875), Origenis Hexaplorum quae supersunt; sive veterum interpretum graecorum in totum Vetus Testamentum fragmenta, Tome II/Texte établi par Field, Clarendoniano, p. 381-405.

Fischer, J. (1986), Song of Songs, Ruth, Lamentations, Ecclesiastes, Esther, Collegeville, The Liturgical Press (Collegeville Bible Commentary 24).

Fischer, A. A. (I997), Skepsis oder Furcht Gottes? Studien zur Komposition und Theologie des Buches Kohelet, Berlin/New York, Walter de Gruyter (Beihefte zur Zeitschrift für die alttestamentliche Wissenschaft 247).

Fischer, S. (1999), Die Aufforderung zur Lebensfreude im Buch Kohelet und seine Rezeption der ägyptischen Harfnerlieder, Francfort-sur-Main, Peter Lang (Wiener Altestamentliche Studien 2).

Foresti, F. (1980), “' 'āmāl in Koheleth: 'Toil' or 'Profit' », Ephemerides Carmeliticae, 31, p. 415-430.

Fox, M. V. (1999), A Time to Tear Down \& a Time to Build Up. A Rereading of Ecclesiastes, Grand Rapids, Eerdmans.

Fox, M. V. (2004), Ecclesiastes, Philadelphia, Jewish Publication Society.

FredericKs, D. C. (1989), "Chiasm and Parallel Structure in Qoheleth 5 : 9-6: 9», Journal of Biblical Literature, 108, p. 17-35.

Fredericks, D. C. (2010), Ecclesiastes, Downers Grove, Inter Varsity Press (Apollos Old Testament Commentary 16).

Galling, K. (1969), Der Prediger, Tübingen, Mohr (Handkommentar zum Alten Testament 1,18). 
Garrett, D. A. (1993), Proverbs. Ecclesiastes. Song of Songs, Nashville, Broadman Press (The New American Commentary 14).

Ginsberg, H. L. (1960), "The Structure and Contents of the Book of Koheleth", dans M. Noth et D. Winton Thomas, dir., Wisdom in Israel and in the Ancient Near East. Presented to Professor Harold Henry Rowley, Leiden, Brill (Supplement to Vetus Testamentum 3), p. 138-149.

Glasser, É. (1970), Le procès du bonheur par Qohélet, Paris, Cerf (Lectio Divina 61).

Goldman, Y. A. P. (2004), "Qoheleth", dans Biblia Hebraica Quinta editione cum apparatu critico novis curis eleborato. General Introduction and Megilloth, Stuttgart, Deutsche Bibelgesellschaft, p. 25-53 et 64-112.

Gordis, R. (19713), Koheleth. The Man and his World, New York, Schocken Books.

Hertzberg, H. W. (1963), Der Prediger, Güttersloh, G. Mohn.

Jérôme (1959), S. Hieronymi Presbyteri Opera. Pars I,1, Turnholti, Brepols (CCSL 72).

Isaksson, B. (1987), Studies in the Language of Qoheleth with Special Emphasison the Verbal System, Stockholm, Almqvist \& Wiksell (Studia Semitica Upsaliensia 10).

Joüon, P. (1982), Grammaire de l'hébreu biblique, Rome, Institut biblique pontifical.

Kamano, N. (2002), Cosmology and Character. Qoheleth's Pedagogy from a Rhetorical-Critical Perspective, Berlin, Walter de Gruyter (Beihefte zur Zeitschrift für die alttestamentliche Wissenschaft 312).

Kamenetzky, A. S. (1904), "Die P'šita zu Kohelet textkritisch und in ihren Verhältnis zu dem massoretischen Text, der Septuagint und den andern alten griechisern Versionen ", Zeitschrift für die alttestamentliche Wissenschaft, 24, p. 181-239.

KRÜGER, T. (1996), "Dekonstruktion und Rekonstruktion prophetischer Eschatologie im Qohelet-Buch", dans A. A. Diesel et al., dir., "Jedes Ding hat seine Zeit...”. Studien zur israelitischen und altorientalischen Weisheit. Diethelm Michel zum 65 Geburstag, Berlin, Walter de Gruyter (Beihefte zur Zeitschrift für die alttestamentliche Wissenschaft 241), p. 107-129.

KRÜGER, T. (1997), «Das Gute und Güter. Erwägungen zur Bedeutung von twb und twbh im Qoheletbuch", Theologische Zeitschrift, 53, p. 53-63. 
Krüger, T. (2004), Qoheleth. A Commentary, Minneapolis, Fortress Press (Hermeneia).

Lane, D. J. (1979), The Old Testament in Syriac According to the Peshitta Version, Part II, Fascicule 5, Leiden, Brill.

LANGe, A. (1991), Weisheit und Torheit bei Kohelet und in seiner Umwelt, Francfort-sur-Main, Bern, Peter Lang (Europäische Hochschulschriften, Reihe XXIII Theologie, Band 433).

Lauha, A. (1978), Kohelet, Neukirchen, Neukirchener Verlag (Biblischer Kommentar. Altes Testament 19).

LAURENT, F. (2002), Les biens pour rien en Qohéleth 5,9-6,6 ou la traversée d'un contraste, Berlin/New York, Walter de Gruyter (Beihefte zur Zeitschrift für die alttestamentliche Wissenschaft 323).

Lavoie, J.-J. (1992), "À quoi sert-il de perdre sa vie à la gagner? Le repos dans le Qohélet », Science et Esprit 44, p. 331-347.

(2004), «amal» et "anah II ", dans Nouveau vocabulaire biblique, Paris/Montréal, Bayard/Médiaspaul, p. 61-65 et 69-79.

(2006), "Habēl habātìm hakol hābel. Histoire de l'interprétation d'une formule célèbre et enjeux culturels ", Science et Esprit, 58, p. 219249.

(2007), «Activité, sagesse et finitude humaine. Étude de Qohélet 1,12-18 ", Laval théologique et philosophique, 63, p. 87-111.

(2008), «Ironie et ambiguïtés en Qohélet 4,13-16 », dans Sciences Religieuses Studies in Religion, 37, p. 15-39.

(2011), «Pouvoir divin et finitude humaine selon Qohélet 6,1-2 », Theoforum, 42, p. 165-188.

(2013), «Ambigüité et pouvoir politique en Qohélet 10,4-7», Rivista Biblica, 61, p. 375-407.

2014a), «Le sage et l'insensé. Étude de Qohélet 7,5-7 », Revue des sciences religieuses, 88, p. 1-26.

(2014b), «Ironie et ambiguités en Qohélet 10,2-3», Science et Esprit, 66, p. 59-84.

Loader, J. A. (1979), Polar Structures in the Book of Qohelet, Berlin, Walter de Gruyter (Beihefte zur Zeitschrift für die alttestamentliche Wissenschaft 152).

L'Hour, J., Borel, M. et Roubaud, J. (2001), «Paroles de Qohélet », dans La Bible. Nouvelle traduction, Paris/Montréal, Bayard/Médiaspaul, p. 1633-1666. 
Loewenklau, L. von (1986), "Kohelet und Sokrates - Versuch eines Vergleiches ", Zeitschrift für die alttestamentliche Wissenschaft, 98, p. 327-338.

Lohfink, N. (1980), Kohelet, Stuttgart, Echter Verlag (Neue Echter Bibel).

Longman III T. (1998), The Book of Ecclesiastes, Grand Rapids/Cambridge, Eerdmans.

Luzzatto, A. (2011), Chi era Qohelet? Traduzione e commento, Brescia, Morcelliana (Pellicano Rosso 135).

Maillot, A. (1987), Qohélet ou l'Ecclésiaste ou La Contestation, Condésur-Noireau, Les bergers et les mages.

Michaud, R. (1987), Qohélet et l'hellénisme. La littérature de Sagesse. Histoire et théologie, II Paris, Cerf (Lire la Bible 77).

Michel, D. (1989), Untersuchungen zur Eigenart des Buches Qohelet Berlin/New York, Walter de Gruyter (Beihefte zur Zeitschrift für die alttestamentliche Wissenschaft 183).

Miller, D. B. (1998), «Qohelet's Symbolic Use of hbl», Journal of Biblical Literature, 117, p. 437-454.

Muilenberg, J. (1954), "A Qoheleth Scroll from Qumran", Bulletin of the American Schools of Oriental Research, 135, p. 20-28.

Murphy, R. E. (1992), Ecclesiastes, Dallas, Word Books (Word Biblical Commentary, 23A).

Ogden, G. S. (1987), Qoheleth, Sheffield, JSOT Press.

Pinçon, B. (2011), Qohélet. Le parti pris de la vie, Partis, Cerf /Médiaspaul (Lire la Bible 169).

Pinker, A. (2009), "Qohelet 6:9 - It looks Better than Tastes ", Journal of Jewish Studies, 60, p. 214-225.

Podechard, E. (1912), L’Ecclésiaste, Paris, Gabalda.

Puech, É. (2001), "Qohelet a Qumran », dans G. Bellia et A. Passaro, dir., Il libro del Qohelet. Tradizione, redazione, teologia, Milano, Paoline (Cammini nello Spirito Biblica 44), p. 144-170.

Rahlfs, A. (1935), Septuaginta, Stuttgart, Deutsche Bibelstiftung.

Rankin, O. S. (1956), "Ecclesiastes", The Interpreter's Bible, Vol. V. Nashville, Abingdon Press, p. 3-88.

Reichert, V. E. et Cohen, A. (I946), "Qhlt. Ecclesiastes", The Five Megilloth, London, The Soncino Press, p. 102-191. 
Reinert, A. (2010), Die Salomofiktion. Studien zu Struktur und Komposition des Koheletbuches, Neukirchen/Vluyn, Neukirchener Verlag (Wissenschaftliche Monographien zum Alten und Neuen Testament 126).

Rose, M. (1999), Rien de nouveau. Nouvelles approches du livre de Qohéleth. Avec une bibliographie (1988-1998) élaborée par Béatrice Perragaux Allisson, Fribourg/Göttingen, Vandenhoeck \& Ruprecht (Orbis Biblicus et Orientalis 168).

Schoors, A. (2004), The Preacher Sought to Find Pleasing Words. A Study of the Language of Qoheleth. Part II. Vocabulary, Leuven, Peeters (Orientalia Lovaniensia Analecta 143).

Schwienhorst-Schönberger, L. (2004), Kohelet, Freiburg (Herders Theologischer Kommentar zum Alten Testament).

Scippa, V. (2010), Qoèlet. L'" arcano progetto di Dio e la gioia della vita", Padova, Edizioni Messaggero (Dabar - Logos - Parola).

ScotT, R. B. Y. (1965), Proverbs. Ecclesiastes Garden City, Doubleday (The Anchor Bible 18).

SEow, C. L. (1997), Ecclesiastes. A New Translation with Introduction and Commentary, New York, Doubleday (Anchor Bible 18c).

Steinmann, J. (1955), Ainsi parlait Qohélet, Paris, Cerf (Lire la Bible 38).

Stendebach, F. J. (2001), "'ayin", Theological Dictionary of the Old Testament, Grand Rapids, Eerdmans, p. 28-44.

STEPHANus, I. (1993), "Qohelet", Revista de interpretacion biblica latinoamericana 15 , p. 75-85.

Strobel, A. (1967), Das Buch Prediger, Düsseldorf, Patmos-Verlag (Die Welt der Bibel. Kleinkommentare zur Heiligen Schrift 9).

Tamez, E. (2000), When the Horizons Close. Rereading Ecclesiastes/trad. par M. Wilde, New York, Maryknoll.

Vilchez Lindez, J. (1994), Sapienciales III. Ecclesiastes o Qobelet, Navarra, Editorial Verbo Divino.

Vinel, F. (2002), La Bible d'Alexandrie. L'Ecclésiaste, Paris, Cerf (La Bible d'Alexandrie 18).

WAHL, J.-J. (2011), Illusion des illusions. Une nouvelle traduction de l'Ecclésiaste, Paris, Desclée de Brouwer (Les carnets).

Walton, T. L. (2006), Experimenting with Qohelet. A Text-Linguistic Approach to Reading Qohelet as Discourse, Maastricht, Uitgeverij Shaker Publishing (Amsterdamse cahiers voor Exegese van de Bijbel en zijn tradities). 
Weeks, S. (2012), Ecclesiastes and Scepticism, New York, T. \& T. Clark (Library of Hebrew Bible/Old Testament Studies 541).

Whitley, C. (I979), Koheleth. His Language and Thought, Berlin, Walter de Gruyter, (Beihefte zur Zeitschrift für die alttestamentliche Wissenschaft 148).

Whybray, R. N. (1981), "The Identification and Use of Quotations in Ecclesiastes", dans J. A. Emerton, dir., Congress Volume. Vienna 1980, Leiden, Brill (Vetus Testamentum Supplement 32), p. 435-451.

Whybray, R. N. (1989), Ecclesiastes, Grand Rapids, Eerdmans.

Yerushalmi, S. (1988), The Book of Koheleth. MeAm Lo'ez,/trad. et adapté par Z. Faier, New York, Moznaim Publishing Corporation.

Zimmerli, W. (1980), Das Buch des Predigers Salomo, Göttingen, Vandenhoeck \& Ruprecht (Das Alte Testament Deutsch 16/1,2).

Zimmerman, F. (1973), The Inner World of Qohelet (With Translation and Commentary), New York, Ktav.

\section{Résumé}

L'auteur présente un état de la recherche des critiques textuelle et des sources de Qohélet 6,7-9 et propose une analyse structurelle et littéraire de ces versets. Puis, il poursuit son enquête en montrant que les ambiguïtés de Qohélet 6,7-9 ne sont pas purement accidentelles. Au contraire, elles font partie d'une stratégie rhétorique qui vise à inciter le lecteur à éprouver différentes lectures.

\section{Abstract}

The author presents the state of research about textual and source criticisms concerning Qohelet 6,7-9, and then provides a structural and literary analysis of these verses. He pursues his inquiry further, showing that the ambiguities of Qohelet 6,7-9 are not purely accidental. On the contrary, these ambiguities are part of a rhetorical strategy that aims at appealing the reader to test various readings. 